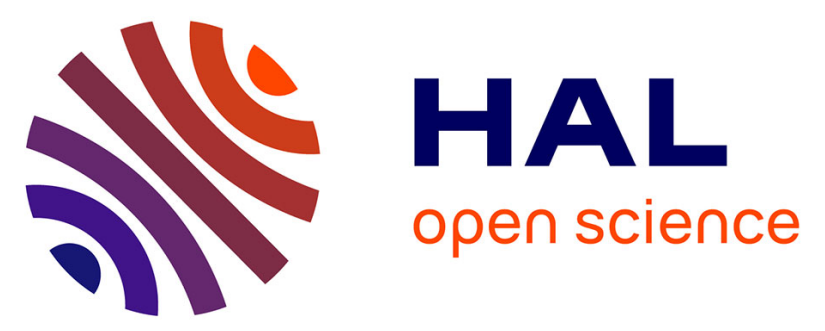

\title{
Two-dimensional modeling of an aircraft engine structural bladed disk-casing modal interaction
}

Mathias Legrand, Christophe Pierre, Patrice Cartraud, Jean-Pierre Lombard

\section{To cite this version:}

Mathias Legrand, Christophe Pierre, Patrice Cartraud, Jean-Pierre Lombard. Two-dimensional modeling of an aircraft engine structural bladed disk-casing modal interaction. Journal of Sound and Vibration, 2009, 319 (1-2), pp.366-391. 10.1016/j.jsv.2008.06.019 . hal-00328186v2

\section{HAL Id: hal-00328186 \\ https://hal.science/hal-00328186v2}

Submitted on 11 Nov 2008

HAL is a multi-disciplinary open access archive for the deposit and dissemination of scientific research documents, whether they are published or not. The documents may come from teaching and research institutions in France or abroad, or from public or private research centers.
L'archive ouverte pluridisciplinaire HAL, est destinée au dépôt et à la diffusion de documents scientifiques de niveau recherche, publiés ou non, émanant des établissements d'enseignement et de recherche français ou étrangers, des laboratoires publics ou privés. 


\title{
Two-dimensional modeling of an aircraft engine structural bladed disk-casing modal interaction
}

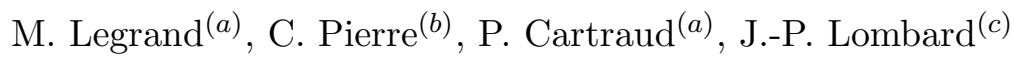 \\ (a) GéM, École Centrale de Nantes, 1 rue de la Noë 44321 Nantes Cedex 3, France \\ (b) Faculty of Engineering, McGill University, MacDonald Engineering Building, 817 \\ Sherbrooke Street West, Room 382, Montreal, Quebec, Canada H3A 2K6 \\ (c) Snecma - Safran group, 77550 Moissy-Cramayel, France
}

\begin{abstract}
In modern turbo machines such as aircraft jet engines, structural contacts between the casing and bladed disk may occur through a variety of mechanisms: coincidence of vibration modes, thermal deformation of the casing, rotor imbalance due to design uncertainties to name a few. These nonlinear interactions may result in severe damage to both structures and it is important to understand the physical circumstances under which they occur. In this study, we focus on a modal coincidence during which the vibrations of each structure take the form of a $k$-nodal diameter traveling wave characteristic of axi-symmetric geometries. A realistic two-dimensional model of the casing and bladed disk is introduced in order to predict the occurrence of this very specific interaction phenomenon versus the rotation speed of the engine. The equations of motion are solved using an explicit time integration scheme in conjunction with the Lagrange multiplier method where friction is accounted for. This model is validated from the comparison with an analytical solution. The numerical results show that the structures may experience different kinds of behaviors (namely damped, sustained and divergent motions) mainly depending on the rotational velocity of the bladed disk.
\end{abstract}

Keywords: contact dynamics, traveling waves, modal interaction, explicit time marching techniques, Lagrange multipliers

\section{Introduction}

In rotordynamics, nonlinear coupling forces between the rotating and surrounding stationary parts can result in unexpected significant displacements and subsequent high stresses leading to structural failure. More specifically in aircraft engines, several mechanisms can contribute to such rotor-to-stator interactions and are usually classified in three main categories:

1. interacting forces due to variations of fluid pressure without structural contact.

2. interacting forces reduced to a unique contacting point along the circumference of both structures.

3. interacting forces induced by multiple simultaneous contacting points at different locations along the circumference.

The first category comprises phenomena such as stall flutter, forced response due to aerodynamic surroundings or acoustic resonance [1]. This category is out of the scope of the present paper. The second category is more or less well understood. The related works usually analyze the vibrations of a rotating shaft with a non-uniform cross-section supported by journal bearings 
where different levels of nonlinearity are considered : oil-film pressure field implicating nonlinear hydrodynamic equations $[2,3]$, direct rub and friction forces, viscous damping forces, nonconstant angular velocity to name a few. These studies mainly necessitate small models with a few coupled nonlinear second-order differential equations suitable for the investigation of a real shaft behavior. Nonlinear and chaotic behaviors as different as dry whip, oil whip or whirling motion are highlighted $[4,5]$. On the other hand, the third category is an emerging field of research and is more specific to aircraft engines like the one depicted in Fig. 1. By virtue of the need of high machine efficiency, it became apparent that more realistic descriptions of fully flexible structures, principally bladed disks and outer casings, within a contact mechanic framework, was required. This efficiency, simply defined as the ratio of energy output to energy input, strongly depends on the clearance between the rotating and stationary components: the wider the clearance, the less efficient the machine. Higher efficiency is achieved by reducing this tip clearance in order to avoid aerodynamic losses. Unfortunately, an obvious consequence is a significant increase in the possibility of rub between the two components with origins such as: gyroscopic effect under certain operating conditions, maneuvering loads during take-off and landing of the aircraft, apparition of a rotor imbalance due to design uncertainties, bird strikes or blade-off, vibrations due to aerodynamic excitations, outer casing distortion caused by a temperature gradient. Depending on the nature of the induced contact, theses interactions can give rise to either (1) very short and transient dynamic responses encountered in crash analysis for example or (2) long lived phenomena characterized by initially intermittent soft contacts that can lead to the excitation of the mode shapes of the structures [6], undesirable large amplitudes and very high stress levels into the structures. In this paper, our emphasis will be in a better

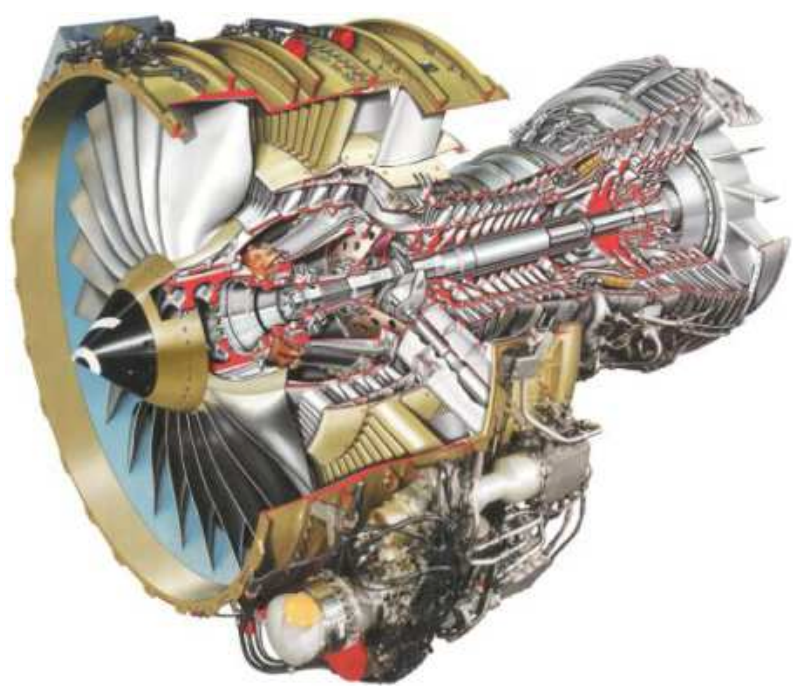

Figure 1: An industrial jet-engine. Courtesy of Snecma, SAFRAN Group

understanding of a very specific kind of contact interaction that can occur in aircraft engines and which belongs to the second class mentioned above. It has been named "traveling wave speed coincidence" in a pioneering work [7] dealing with this phenomenon and rich in insight. This very-well documented research includes an extensive bibliography on rotordynamics in general, addresses the aforementioned problem in which an analytical condition of interaction is determined [8], and suggests a solution method. Finally, experimental and numerical results are compared and show good agreement. New results were given in [9] and the present paper provides further developments. 
Starting from simple considerations on structures that are rotationally periodic or exhibit axi-symmetry, it is proved that $k$-nodal diameter rotating modes (see Appendix A for discussion) that separately propagate in the bladed disk and casing, may interact through direct contact due to the small tip clearance. This modal interaction (or traveling wave speed coincidence as suggested in [7]) can be quickly described as a geometric matching between these traveling $k$-nodal diameter modes, forward in the casing and counter-rotating in the bladed disk (see Appendix A for the explanation of these directional terms), that display the same absolute traveling velocity due to the rotation of the bladed disk. As a combination of self-excited mode shapes, high tangential velocity of the blade-tips (from 400 to $600 \mathrm{~m} / \mathrm{s}$ ) and stabilizing or destabilizing rub [10], this nonlinear interaction may lead to devastating effects like the destruction of the engine. It is therefore important to get a better description of the phenomenon and give accurate predictions of the behavior with respect to a series of mechanical parameters of interest. To this end, a numerical tool, based on two-dimensional elastic outer casing and bladed disk, has been developed. It combines a low computational cost with a fairly realistic modeling. Each structure is represented in terms of its two $k$-nodal diameter vibration modes which allow for traveling wave motions to occur. The kinetic energy of the bladed disk is transformed into vibratory energy through direct contact and may result in a case of interaction. In this numerical tool, in conjunction with the Lagrange multiplier method where friction is accounted for, the governing equations are solved using a time-stepping method based on the explicit central differences scheme more relevant to transitional dynamics dealing with contact constraints [11]. Attention is given to a set of parameters such as: angular velocity of the rotor, friction coefficient, number of nodal diameters, number of blades, initial curvature of the blades. Comparison with analytical derivation is also provided.

\section{Modal interaction definition}

This section presents the interaction phenomenon of interest which can only occur for a certain class of structures featuring cyclic-symmetry and/or axi-symmetry.

\subsection{Cyclic symmetry}

A reference sector is defined as a blade plus the corresponding segment of the disk. An aircraft engine bladed disk composed of $N$ sectors is obtained by $N-1$ repeated rotations of such a reference sector through the fundamental interblade phase shift angle $\alpha=2 \pi / N$. The $N$ identical sectors form a perfectly tuned circular system said to show rotational periodicity or cyclic symmetry [12]. Typically, the finite element analysis of such a structure results in circulant mass and stiffness matrices. The associated eigenproblem in physical coordinates $\mathbf{u}$ is written as [13]

$$
\mathbf{Y u}=\mathbf{0}
$$

with

$$
\mathbf{Y}=\left[\begin{array}{cccccc}
\mathbf{Y}_{0} & \mathbf{Y}_{1} & \mathbf{0} & \cdots & \mathbf{0} & \mathbf{Y}_{1}^{T} \\
\mathbf{Y}_{1}^{T} & \mathbf{Y}_{0} & \mathbf{Y}_{1} & \mathbf{0} & \cdots & \mathbf{0} \\
& \ddots & \ddots & \ddots & & \\
& & \ddots & \ddots & \ddots & \\
\mathbf{0} & \cdots & \mathbf{0} & \mathbf{Y}_{1}^{T} & \mathbf{Y}_{0} & \mathbf{Y}_{1} \\
\mathbf{Y}_{1} & \mathbf{0} & \cdots & \mathbf{0} & \mathbf{Y}_{1}^{T} & \mathbf{Y}_{0}
\end{array}\right]
$$


In Eq.(2), the number of blocks on the diagonal is equal to the number of sectors and $\mathbf{Y}_{i}=$ $\mathbf{K}_{i}-\omega^{2} \mathbf{M}_{i}$ for $i=[0,1]$. The stiffness matrix $\mathbf{K}_{0}$ and the mass matrix $\mathbf{M}_{0}$ are derived from the finite element model of a fundamental sector, while matrices $\mathbf{M}_{1}$ and $\mathbf{K}_{1}$ are built by considering displacement compatibility between adjacent sectors. In the context of symmetrical components within block-circulant matrix theory, it can be shown that the projection of $\mathbf{Y}$ onto the realcyclic coordinates is block diagonal [14]. Each block is associated with a parameter $k$ and has the partitions

$$
\tilde{\mathbf{Y}}^{k}=\left[\begin{array}{cc}
\mathbf{Y}_{0}+\mathbf{Y}_{2} \cos k \alpha & \mathbf{Y}_{3} \sin k \alpha \\
-\mathbf{Y}_{3}^{T} \sin k \alpha & \mathbf{Y}_{0}+\mathbf{Y}_{2} \cos k \alpha
\end{array}\right],
$$

where $\mathbf{Y}_{2}=\mathbf{Y}_{1}+\mathbf{Y}_{1}^{T}$ and $\mathbf{Y}_{3}=\mathbf{Y}_{1}-\mathbf{Y}_{1}^{T}$. Consequently, the initial eigenvalue problem (1) reduces to a series of smaller eigenvalue problems mostly depending on $k$. By noting $\mathbf{x}$, the counterpart of the physical displacement vector $\mathbf{u}$ in real-cyclic coordinates, the series of new eigenvalue problems becomes ( $k=0$ is not of interest to our study)

$$
\widetilde{\mathbf{Y}}^{k} \mathbf{x}^{k}=\mathbf{0}, k=1, \ldots, K \text { with } K= \begin{cases}\frac{N-1}{2} & \text { if } N \text { is odd } \\ \frac{N-2}{2} & \text { if } N \text { is even. }\end{cases}
$$

The skew-symmetric structure of $\widetilde{\mathbf{Y}}^{k}$ gives rise to mode pairs with identical natural frequencies [15]. By partitioning the eigenvector matrix as $\mathbf{x}^{k}=\left[\mathbf{x}^{\mathbf{c}, k}, \mathbf{x}^{\mathbf{s}, k}\right]^{T}$, both respective modes can be expressed as

$$
\mathbf{x}_{\omega, 1}^{k}=\left[\begin{array}{c}
\mathbf{x}^{\mathbf{c}, k} \\
\mathbf{x}^{\mathbf{s}, k}
\end{array}\right]_{\omega, 1}=\left(\begin{array}{c}
\mathbf{x}_{\omega, 1}^{\mathbf{c}, k} \\
\mathbf{x}_{\omega, 1}^{\mathbf{s}, k}
\end{array}\right) \text { and } \mathbf{x}_{\omega, 2}^{k}=\left[\begin{array}{c}
\mathbf{x}^{\mathbf{c}, k} \\
\mathbf{x}^{\mathbf{s}, k}
\end{array}\right]_{\omega, 2}= \pm\left(\begin{array}{c}
-\mathbf{x}_{\omega, 1}^{\mathbf{s}, k} \\
\mathbf{x}_{\omega, 1}^{\mathbf{c}, k}
\end{array}\right) .
$$

Their shapes are similar and rotated around the axis of symmetry by $\pi /(2 k)$. Once known

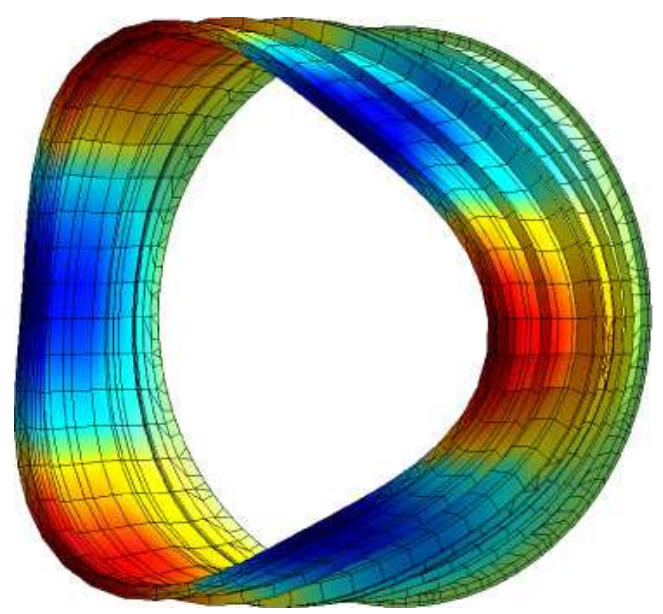

(a)

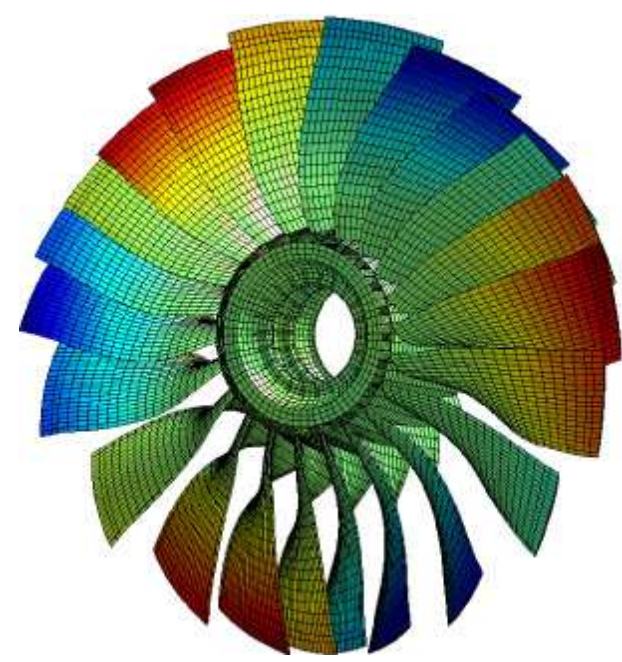

(b)

Figure 2: 3-nodal diameter modes of (a) a jet engine casing and (b) a bladed disk

in real-cyclic coordinates, they can be transformed back into physical coordinates for the $n^{\text {th }}$ sector by using

$$
\mathbf{u}_{n}=\sqrt{\frac{2}{N}} \sum_{k=1}^{K}\left[\mathbf{x}^{\mathbf{c}, k} \cos (n-1) k \alpha+\mathbf{x}^{\mathbf{s}, k} \sin (n-1) k \alpha\right]+\frac{1}{\sqrt{N}} \mathbf{x}^{0}+\frac{(-1)^{n-1}}{\sqrt{N}} \mathbf{x}^{\frac{N}{2}} .
$$


In Eq. (6), the last term exists only if there is an even number of sectors. This equation provides a geometrical interpretation of $k$, or number of nodal diameters depicted in Fig. 2 for $k=3$ : a sinusoidal repartition of the displacements along each spatial direction.

Both shapes of sector $n$ along the $k$-nodal diameter mode pair are given in physical coordinates by combining Eqs. (5) and (6) to get

$$
\begin{aligned}
& \mathbf{u}_{n}^{1}=\mathbf{x}^{\mathbf{c}, k} \cos (n-1) k \alpha-\mathbf{x}^{\mathbf{s}, k} \sin (n-1) k \alpha, \\
& \mathbf{u}_{n}^{2}=\mathbf{x}^{\mathbf{s}, k} \cos (n-1) k \alpha+\mathbf{x}^{\mathbf{c}, k} \sin (n-1) k \alpha .
\end{aligned}
$$

It is then possible to combine these two modes into forward $\left({ }^{f}\right)$ and backward $\left({ }^{b}\right)$ rotating modes at angular frequency $\omega$ by multiplying by the appropriate time-dependent functions

$$
\begin{aligned}
& \mathbf{u}_{n}^{f}(t)=\mathbf{u}_{n}^{1} \cos \omega t+\mathbf{u}_{n}^{2} \sin \omega t, \\
& \mathbf{u}_{n}^{b}(t)=\mathbf{u}_{n}^{1} \cos \omega t-\mathbf{u}_{n}^{2} \sin \omega t .
\end{aligned}
$$

The group velocity of such waves, which is of great interest in this work, is well known to be the angular frequency $\omega$ divided by the wavenumber $k$ (or number of nodal diameters in our case)

$$
\nu=\frac{\omega}{k} \text {. }
$$

\subsection{Axi-symmetry}

Structures featuring perfect axi-symmetry, such as outer casings, can be viewed as limit cases of rotationally periodic structures: the number of sectors tends to infinity. Therefore, they share the same properties and have degenerate orthogonal pairs as well [16]. These mechanical characteristics are intrinsically connected to the structural invariance with respect to the revolution axis of their geometric shape. Any mode can be subjected to a rotation and is necessarily a linear combination of two orthogonal "fundamental" modes with similar shape and equal frequency.

As an important consequence, any linear displacement of a structure featuring cyclic-symmetry or perfect axi-symmetry can be seen as a linear combination of $k$-nodal diameter forward and backward rotating modes [12].

\subsection{Interaction phenomenon: an analytical approach}

Coming back to Eq. (9), the group velocity of a $k$-nodal diameter rotating mode propagating in a motionless structure is given by $\pm \omega / k$ in a stationary frame, depending on the direction of propagation. Accordingly, for a rotating structure observed in a stationary reference frame, the rotational velocity $\Omega$ (selecting the counter-clockwise direction as positive) has to be accounted for. The propagation velocity becomes then $\Omega+\omega / k$ for the co-rotating mode and $\Omega-\omega / k$ for the counter-rotating mode. Frequencies of rotating structures are usually $\Omega$-dependent due to centrifugal stiffening. In our study, this centrifugal stiffening is neglected.

In the following, the two natural frequencies respective to the chosen double modes are denoted $\omega_{c}$ for the casing and $\omega_{b d}$ for the bladed disk.

For a certain angular velocity of the bladed disk, the rotating modes in the bladed disk may travel at the same absolute speed as the forward rotating mode in the casing. Furthermore, if both structures vibrate at their own natural frequency, this certain angular velocity becomes a critical velocity for which two cases of traveling wave coincidence (equally said rotating mode coincidence) can be stated as plotted in Fig. 3, in other words

$$
\frac{\omega_{c}}{k}=\Omega \pm \frac{\omega_{b d}}{k} .
$$




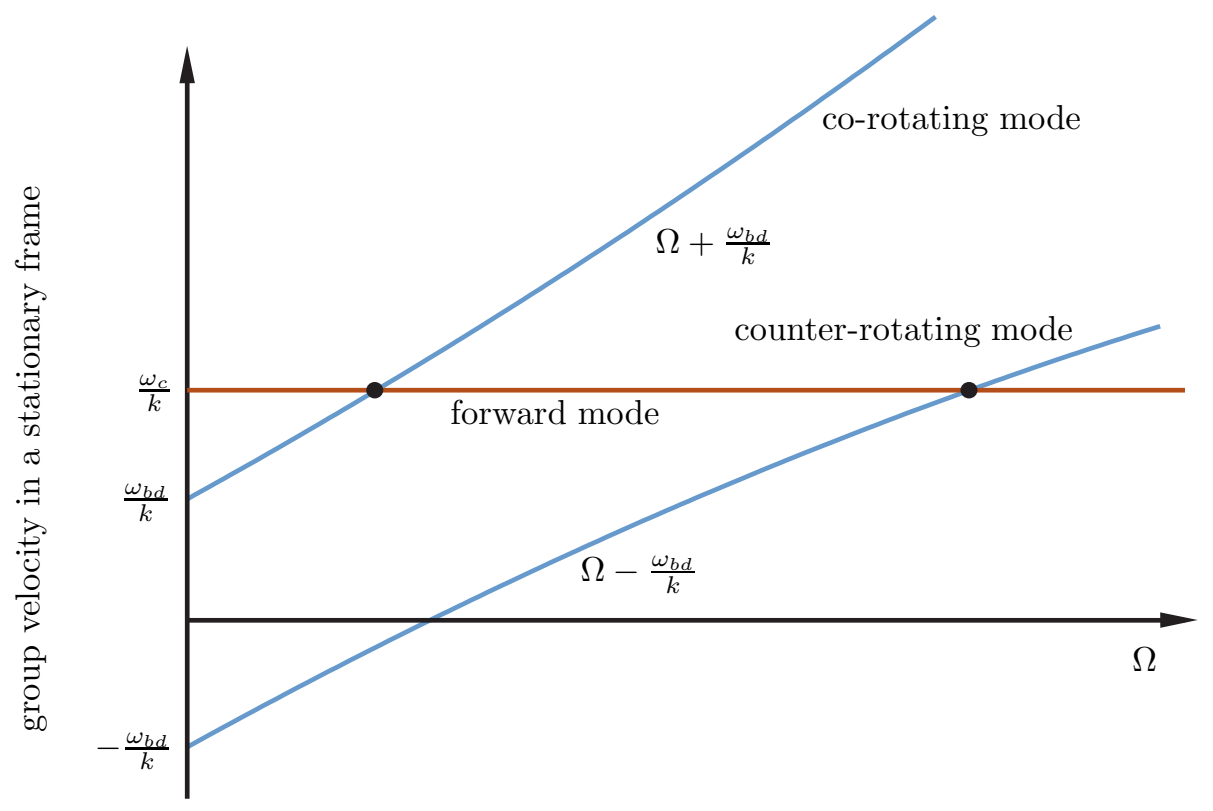

Figure 3: Theoretical modal interaction conditions • as an extension of a Campbell diagram for $k$-nodal diameter modes. The frequency $\omega_{c}$ is usually greater than $\omega_{b d}$ of the first flexural mode of the blades. Both lines of equation $\Omega \pm \omega_{b d} / k$ are normally curved as $\omega_{b d}$ is $\Omega$-dependent: in our study, they are considered as straight lines, meaning that no centrifugal stiffening is accounted for.

Taking into account physical considerations on the direction of the contact and friction forces between the two structures - forward in the casing and backward in the bladed disk due to the chosen direction of rotation, and invoking the action/reaction principle of Newton's third law (the contact efforts exerting on the casing will have an opposite direction to their counterparts exerting on the bladed-disk), only one of these equations can be considered as dangerous,

$$
\omega_{c}=k \Omega_{c t}-\omega_{b d}
$$

This condition of interaction shows that there exists a unique critical speed $\Omega_{c t}$ for which, in theory, both structures are driven in resonance by the contact forces. From a stationary frame, the forward rotating mode in the casing propagating at $\omega_{c} / k$ geometrically matches with its counter-rotating counterpart in the bladed disk propagating at $\Omega_{c t}-\omega_{b d} / k$ as shown in a very simple manner in Fig. 4 . Consequently, the angular velocity $\Omega_{c t}$ must be avoided because this bi-resonant phenomenon in $\omega_{c}$ and $\omega_{b d}$ may theoretically cause undesired large amplitude vibrations.

Inevitably, these very simple considerations compared to a fully nonlinear analysis involving contact constraints, although meaningful as a first approach, fail to answer a series of questions dealing with the reduction, not to say the disappearance of this phenomenon:

1. Structural damping role.

2. Effectiveness of an abradable coating between the blade tips and the outer casing.

3. Influence of the blade design in the occurrence of the phenomenon.

4. Efficiency of intentional mistuning for circumventing this problem by separating mode pairs and, as a consequence, reducing the amount of energy transferred from one component to the other one. 


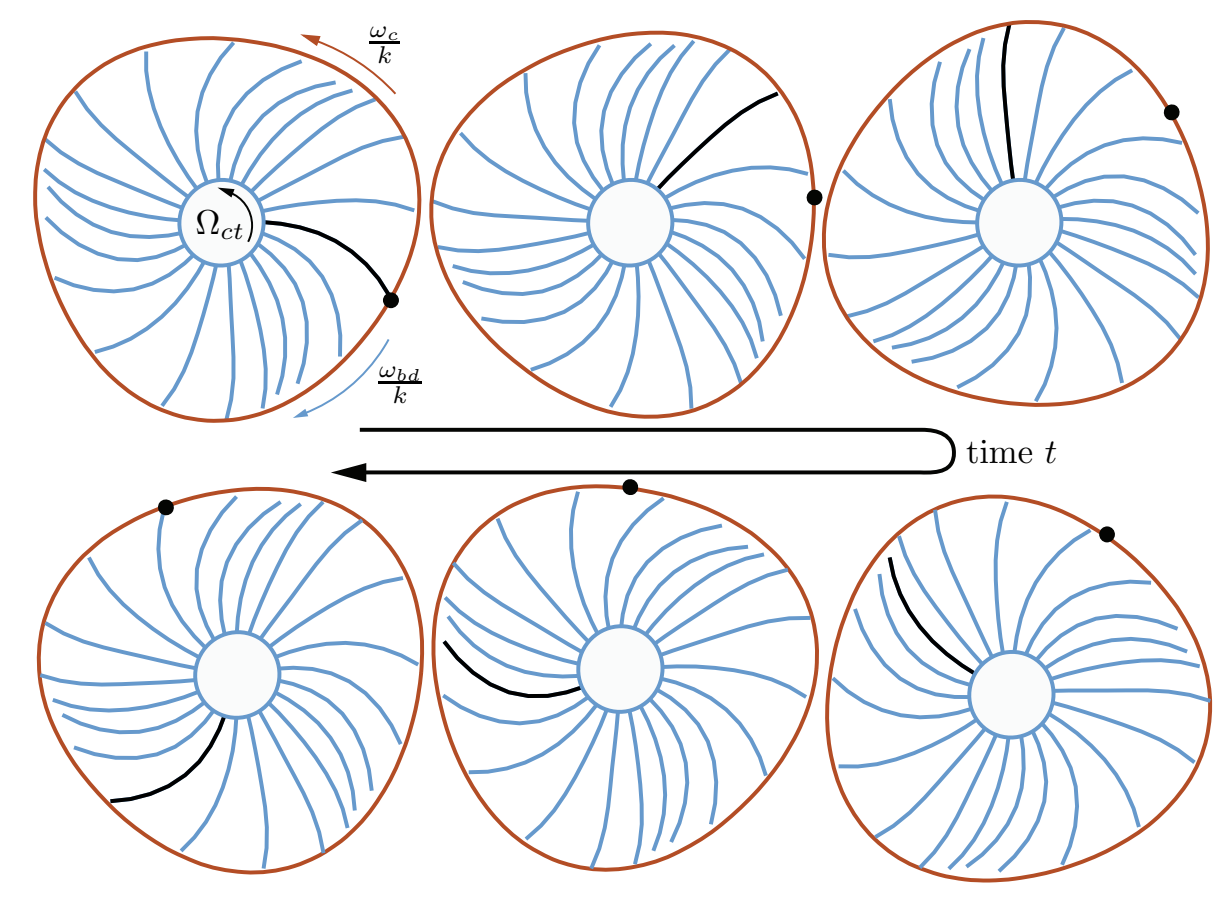

Figure 4: Schematic of a modal interaction coincidence for $k=3$. The bladed disk rotates clockwise at $\Omega_{c t}$ as shows the black blade. From a stationary frame, a forward rotating 3-nodal diameter mode propagates at $\omega_{c} / k$ in the casing (marked by a black dot) and its counter-rotating counterpart at $\Omega_{c t}-\omega_{b d} / k$ in the bladed disk.

More generally, a comprehensive modeling, analysis and understanding is of great interest and critical if we want to isolate the most influential mechanical parameters involved in this interaction phenomenon. Thereby, our main concern is to develop a numerical predicting tool, fast and reliable, suitable for a better insight and ultimately able to give answers to the questions listed above ${ }^{1}$.

\section{Structural models}

Two-dimensional models of a bladed disk and a casing used in our numerical tool are presented in the following. In order to be as much accurate as 3D models, the design of the structures requires (1) both normal contact and friction forces treatment between the casing and blade-tips considering a physical law and (2) the chosen mode shapes of the casing and the bladed disk to geometrically match together. In other words, the displacement field of both structures must be sufficiently rich to allow for normal and bending displacements to be a priori independent unknowns [9].

\subsection{Bladed disk}

The blades are discretized by the usual two-noded Euler-Bernoulli straight beam elements with three degrees of freedom $u_{b l(n)}, v_{b l(n)}$ and $\gamma_{b l(n)}$ per node. In the $v_{b l}$-direction, the local shape functions are

$$
\begin{array}{ll}
N_{1}(x)=1-3 x^{2}+2 x^{3}, & N_{2}(x)=l_{b}\left(x-2 x^{2}+x^{3}\right), \\
N_{3}(x)=3 x^{2}-2 x^{3}, & N_{4}(x)=l_{b}\left(-x^{2}+x^{3}\right) .
\end{array}
$$

\footnotetext{
${ }^{1}$ Only a few of them will be considered in this paper.
} 


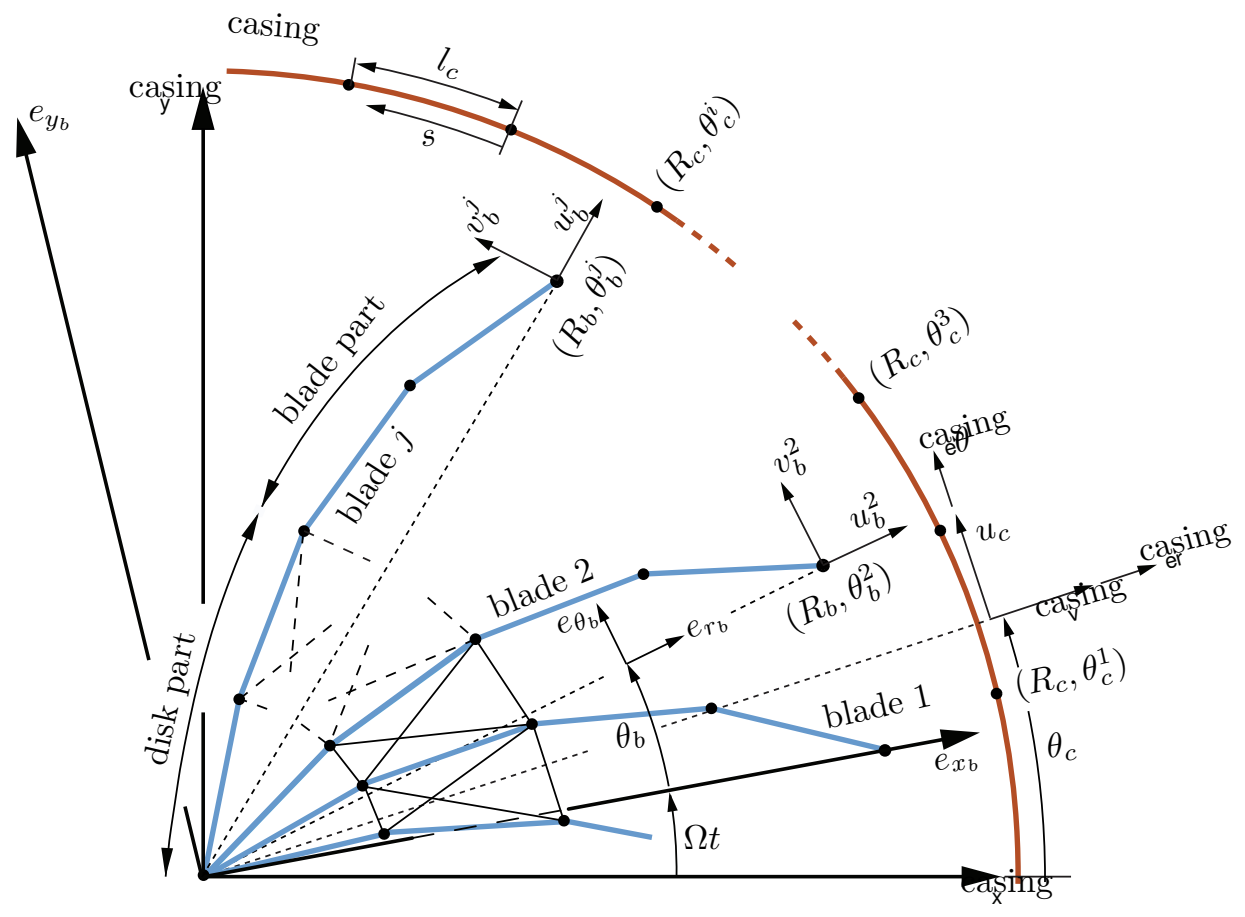

Figure 5: Schematic of the reference configuration of the bladed disk and casing in their respective frame $\left(e_{x_{b}}, e_{y_{b}}\right)$ and $\left(e_{x_{c}}, e_{y_{c}}\right)$.

They are linear along $u_{b l}$ and expressed as

$$
M_{1}(x)=1-x \text { and } M_{2}(x)=x, x \in[0,1] .
$$

In a local frame fixed to a rod-beam finite element, the discretized displacement field of the $i^{\text {th }}$ element is

$$
\begin{aligned}
& u_{b l}^{i}(x)=M_{1}(x) u_{b l(n)}^{i}+M_{2}(x) u_{b l(n)}^{i+1}, \\
& v_{b l}^{i}(x)=N_{1}(x) v_{b l(n)}^{i}+N_{2}(x) \gamma_{b l(n)}^{i}+N_{3}(x) v_{b l(n)}^{i+1}+N_{4}(x) \gamma_{b l(n)}^{i+1} .
\end{aligned}
$$

In Fig. 5, a network of linear springs connects the blades together to model the disk. The coupling between the normal and transversal displacements, respectively $u_{b l}$ and $v_{b l}$, at the blade tip location is achieved by curving the geometry of the blades. This represents the cornerstone of our structural modeling. Indeed, a simple straight blade geometry in its equilibrium position does not meet the second requirement listed above: the mode shapes in $u_{b l}$ and $v_{b l}$ directions would be fully uncoupled rendering a geometric match between the $k$-nodal diameter modes of both the casing and the bladed disk impossible.

Finally, for the sake of simplicity and due to the inherent motion of the bladed disk, all quantities relative to this structure (mainly displacement vectors and mechanical matrices) are projected onto a polar coordinate system with unit vectors $\left(e_{r_{b}}, e_{\theta_{b}}\right)$ rigidly linked to the structure. The displacements of the blade-tip $j$, initially located at the point $\left(R_{b}, \theta_{b}^{j}\right)$ where $R_{b}$ refers to the external radius of the bladed disk, are respectively denoted by $u_{b}^{j}$ along $e_{r_{b}}$ and $v_{b}^{j}$ along $e_{\theta_{b}}$ as illustrated in Fig. 5. The angle $\theta_{b}^{j}$ is defined from $e_{x_{b}}$ rigidly fixed to the first blade and yields

$$
\theta_{b}^{j}=\frac{2 \pi}{N}(j-1), \quad j=1, \ldots, N .
$$




\subsection{Casing}

A polar coordinate system with unit vectors $e_{\theta_{c}}$ and $e_{r_{c}}$ is assigned to the casing. The latter is discretized using two-noded curved beam finite elements where both the displacement fields $u_{c}$ and $v_{c}$ (respectively along $e_{\theta_{c}}$ and $e_{r_{c}}$ ) are assumed cubic polynomials and involve four degrees of freedom per node: $u_{c(n)}, u_{c(n), s}, v_{c(n)}$ and $v_{c(n), s}$ as depicted in Fig. 5. The initial location of the node $i$ is given by the doublet $\left(R_{c}, \theta_{c}^{(i)}\right)$ where $R_{c}$ refers to the radius of the casing. The shape functions are similar to those of the bladed disk, by replacing $x$ by $s$ and $l_{b}$ by the length $l_{c}$ of a finite element of the casing in Eq. (12) where $s \in[0,1]$ is the local path variable. This formulation is taken from [17] where it is shown that such finite elements are locking free. The discretized displacement field of element $i$ whose nodes are denoted $i$ and $i+1$ is then

$$
\begin{aligned}
& u_{c}^{i}(s)=N_{1}(s) u_{c(n)}^{i}+N_{2}(s) u_{c(n), s}^{i}+N_{3}(s) u_{c(n)}^{i+1}+N_{4}(s) u_{c(n), s}^{i+1}, \\
& v_{c}^{i}(s)=N_{1}(s) v_{c(n)}^{i}+N_{2}(s) v_{c(n), s}^{i}+N_{3}(s) v_{c(n)}^{i+1}+N_{4}(s) v_{c(n), s}^{i+1} .
\end{aligned}
$$

\subsection{Modal reduction}

In order to focus on a modal interaction, both components are reduced to their respective two $k$-nodal diameter vibration modes as shown in Fig. 6 . In what follows, $\mathbf{M}, \mathbf{D}$ and $\mathbf{K}$ represent

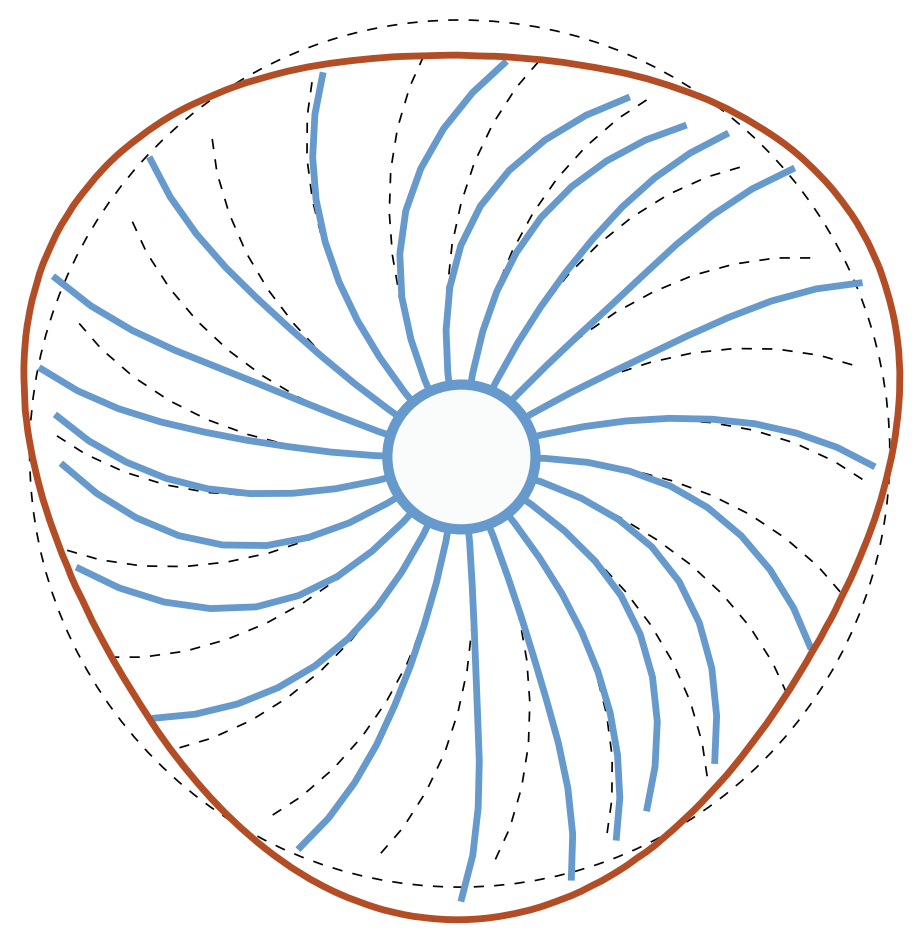

Figure 6: 3-nodal diameter modes of the casing and the bladed disk

respectively the mass, damping and stiffness matrices coming from the discretization in space and include the bladed disk and the casing. Their $4 \times 4(2 \times 2$ for each structure $)$ modal counterparts are denoted $\mathbf{M}_{\mathbf{m}}, \mathbf{D}_{\mathbf{m}}$ and $\mathbf{K}_{\mathbf{m}}$ and related to each other by

$$
\mathbf{M}_{\mathbf{m}}=\mathbf{P}^{T} \mathbf{M P} \text { with } \mathbf{P}=\left[\begin{array}{cc}
\mathbf{P}_{c} & \mathbf{0} \\
\mathbf{0} & \mathbf{P}_{b d}
\end{array}\right]
$$

where $\mathbf{P}_{c}$ (resp. $\mathbf{P}_{b d}$ ) is constituted of the two retained $k$-nodal diameter modes of the casing (resp. bladed disk). By noting $\mathbf{u}$ the vector storing all the physical displacement components 
of both structures, an equivalent expression

$$
\mathbf{u}=\mathbf{P} \mathbf{u}_{\mathbf{m}}
$$

holds. In Eq. (18), $\mathbf{u}_{\mathbf{m}}=\left[u_{m, c}^{1} u_{m, c}^{2} u_{m, b}^{1} u_{m, b}^{2}\right]^{T}$ is the modal counterpart of $\mathbf{u}$. It is worth noting that $u_{m, b}^{1}$ and $u_{m, b}^{2}$ are defined with respect to the rotating frame $\left(e_{x_{b}}, e_{y_{b}}\right)$ whereas $u_{m, c}^{1}$ and $u_{m, c}^{2}$ are defined with respect to the frame $\left(e_{x_{c}}, e_{y_{c}}\right)$.

\section{Contact dynamics}

\subsection{General theory}

The contacting forces in the normal and tangential directions exerted by the blade-tips on the casing and vice versa are of primary interest in this study. Equations of motion are derived using the Principle of Virtual Work within the kinematically linear framework following, in essence, the procedure described in [18].

First, it is convenient to arbitrarily choose one surface subject to contact as the master one so that the second one, commonly called the slave surface, can be parameterized. It is then possible to find for any material point $\mathbf{x}$ belonging to the master surface $\Gamma_{c}^{(m)}$, its closest counterpart $\overline{\mathbf{y}}$ on the slave surface $\Gamma_{c}^{(s)}$

$$
\overline{\mathbf{y}}=\underset{\mathbf{y} \in \Gamma_{c}^{(s)}}{\operatorname{argmin}}\|\mathbf{x}-\mathbf{y}\|,
$$

where $\operatorname{argmin} f(x)$ is the value of $x$ for which the function $f(x)$ reaches its smallest value. According to these notations, the gap function between the two structures becomes

$$
\mathrm{g}(\mathbf{x})=\mathrm{g}_{0}(\mathbf{x})+\left(\mathbf{u}^{(m)}(\mathbf{x})-\mathbf{u}^{(s)}(\overline{\mathbf{y}}(\mathbf{x}))\right) \cdot \mathbf{n},
$$

where $\mathrm{g}_{0}(\mathbf{x})$ represents the initial positive gap between the two structures and $\mathbf{n}$, the outward normal to $\Gamma_{c}^{(s)}$. The contact conditions, referred to as the Kuhn-Tucker optimality conditions in the parlance used in the literature, take the form

$$
\forall \mathbf{x} \in \Gamma_{c}^{(m)}, \quad t_{N} \geq 0, \mathrm{~g} \geq 0, t_{N} \mathrm{~g}=0,
$$

where $t_{N}$ stands for the contact pressure, assumed positive, acting on $\Gamma_{c}^{(s)}$. To these unilateral contact conditions, we add the well-known Coulomb friction law

$$
\begin{aligned}
& \left\|\mathbf{t}_{\mathbf{T}}\right\| \leq \mu t_{N}, \\
& \left\|\mathbf{t}_{\mathbf{T}}\right\|<\mu t_{N} \Rightarrow \mathbf{v}_{\mathbf{T}}=0, \\
& \left\|\mathbf{t}_{\mathbf{T}}\right\|=\mu t_{N} \Rightarrow \exists \alpha>0 \text { such as } \mathbf{v}_{\mathbf{T}}=\alpha \frac{\mathbf{t}_{\mathbf{T}}}{\left\|\mathbf{t}_{\mathbf{T}}\right\|},
\end{aligned}
$$

for which $\mu$ is the coefficient of friction, $\mathbf{v}_{\mathbf{T}}$, the tangential slip rate and $\mathbf{t}_{\mathbf{T}}$, the tangential stress vector. To enforce the so-called impenetrability condition in a very general way, it is necessary to construct an admissible contact pressure field $C_{N}$ and its counterpart relative to friction tractions, $C_{T}\left(t_{N}\right)$ as

$$
\begin{aligned}
& C_{N}=\left\{t_{N}: \Gamma_{c}^{(s)} \rightarrow \mathbb{R} \backslash t_{N} \geq 0\right\}, \\
& C_{T}\left(t_{N}\right)=\left\{\mathbf{t}_{\mathbf{T}}: \Gamma_{c}^{(s)} \rightarrow \mathbb{R}^{3} \backslash \mathbf{t}_{\mathbf{T}} \cdot \mathbf{n}=0,\left\|\mathbf{t}_{\mathbf{T}}\right\| \leq \mu t_{N}\right\} .
\end{aligned}
$$


An alternative equivalent form to Eqs. (21) and (22) is

$$
\begin{aligned}
& \forall s_{N} \in C_{N}, \int_{\Gamma_{c}^{(s)}} \mathrm{g}\left(s_{N}-t_{N}\right) \mathrm{d} \mathrm{S} \geq 0, \\
& \forall \mathbf{s}_{\mathbf{T}} \in C_{T}\left(t_{N}\right), \int_{\Gamma_{c}^{(s)}} \mathbf{v}_{\mathbf{T}} \cdot\left(\mathbf{s}_{\mathbf{T}}-\mathbf{t}_{\mathbf{T}}\right) \mathrm{d} \mathrm{S} \geq 0 .
\end{aligned}
$$

The weak form of the contact problem can be then written in the following manner:

find the admissible displacement field $\mathbf{u}$ such as for all admissible virtual displacement $\boldsymbol{\delta} \mathbf{u}$,

$$
\int_{\Omega} \rho \ddot{\mathbf{u}} \cdot \boldsymbol{\delta} \mathbf{u} \mathrm{dV}+\int_{\Omega} \overline{\overline{\boldsymbol{\sigma}}}: \boldsymbol{\delta} \overline{\bar{\varepsilon}} \mathrm{dV}=\int_{\Gamma_{\sigma}} \mathbf{t}_{\mathbf{d}} \cdot \boldsymbol{\delta} \mathbf{u} \mathrm{dS}+\int_{\Gamma_{c}^{(s)}}\left(t_{N} \delta \mathrm{g}+\mathbf{t}_{\mathbf{T}} \cdot \boldsymbol{\delta} \mathbf{u}_{\mathbf{T}}\right) \mathrm{dS}+\int_{\Omega} \mathbf{f}_{\mathbf{d}} \cdot \boldsymbol{\delta} \mathbf{u} \mathrm{dV}
$$

where $t_{N}$ and $\mathbf{t}_{\mathbf{T}}$ are constrained by conditions (24). The solution of this problem relies on a nonlinear iterative procedure within which the unknown Lagrange multipliers in the normal and tangential directions will have to converge respectively towards $t_{N}$ and $\mathbf{t}_{\mathbf{T}}$.

\subsection{Solution method}

Once discretized in space using the shape functions described above for $\mathbf{u}$ and $\boldsymbol{\delta} \mathbf{u}$, formulation (25) has to be resolved in time. The question concerning the best compromise for solving a dynamic contact problem involving friction and deformable bodies is still open. The difficulty of contact problems emanates from the fact that contact interactions are not only nonlinear but non-smooth as well. On the first hand, a selection has to be made among implicit time-marching techniques, more reliable and rigorous with respect to equilibrium at each time step, and explicit procedures more relevant for transient dynamics dealing with high nonlinearities. On the other hand, the enforcement of the contact constraints has to be tackled by using a penalty regularization [19] or by employing Lagrange multipliers [20] or a combination of the latter ones, the Augmented Lagrangian Method [21]. Other formulations can also be identified [22] and various conclusions concerning their efficiency can be drawn [23]. Consequently, many different computational strategies have been developed over the last few years [24]. As a matter of fact, these methods have to combine a continuous normal evolution of the contact surface with energy momentum conserving algorithms in order to ensure convergence. In our work, the first task is achieved by choosing curved beam finite elements for discretizing the casing that guarantees a continuous second derivative of the normal direction. The second task is completed with the use of the Forward Increment Lagrange Method suggested in [25] associating simplicity of implementation with highly accurate and well-behaved solution. The obtained matrix form of (25) involving $\mathbf{M}, \mathbf{D}$ and $\mathbf{K}$, is solved using the explicit central differences scheme. Denoting the time step by $h$, vectors in velocity and acceleration take the form, respectively

$$
\dot{\mathbf{u}}_{n}=\frac{\mathbf{u}_{n+1}-\mathbf{u}_{n-1}}{2 h} \text { and } \ddot{\mathbf{u}}_{n}=\frac{\mathbf{u}_{n+1}-2 \mathbf{u}_{n}+\mathbf{u}_{n-1}}{h^{2}} .
$$

The solution method is divided into three steps:

1. Prediction of the displacements $\mathbf{u}_{n+1}$ of the ongoing time-step $n$ by ignoring the contact reactions. These predicted displacements $\mathbf{u}_{n+1, p}$ (with subscript ${ }_{p}$ for prediction) are expressed as

$$
\mathbf{u}_{n+1, p}=\left[\frac{\mathbf{M}}{h^{2}}+\frac{\mathbf{D}}{2 h}\right]^{-1}\left(\left(\frac{2 \mathbf{M}}{h^{2}}-\mathbf{K}\right) \mathbf{u}_{n}+\left(\frac{\mathbf{D}}{2 h}-\frac{\mathbf{M}}{h^{2}}\right) \mathbf{u}_{n-1}+\mathbf{F}_{n}^{\mathbf{e x t}}\right) .
$$


2. Determination of the gap function vector $\mathbf{g}_{n+1, p}$ between these structures following Eq. (20). A search algorithm identifies all contactor nodes that have penetrated the target domain. Satisfying the impenetrability conditions implies that the final gap functions $\mathbf{g}_{n+1}$ (linearized when necessary) must be positive or vanish, meaning

$$
\mathbf{g}_{n+1}=\mathbf{C}_{\mathbf{N}}^{T} \mathbf{u}_{n+1, c}+\mathbf{g}_{n+1, p} \geq \mathbf{0},
$$

where the corrected displacements $\mathbf{u}_{n+1, c}$ are being calculated (with subscript ${ }_{c}$ for correction). $\mathbf{C}_{\mathbf{N}}$ is the contact constraint matrix in the normal direction. Eq. (28) is rewritten in an equivalent form

$$
\mathbf{C}_{\mathbf{N}}^{T} \mathbf{u}_{n+1, c}+\mathbf{g}_{n+1, p}^{-}=\mathbf{0}
$$

where $\mathbf{g}_{n+1, p}^{-}=\operatorname{Proj}_{\mathbb{R}_{-}^{\mathrm{Sg}}}\left(\mathbf{g}_{n+1, p}\right)$ and $\mathrm{Sg}=\operatorname{size}\left(\mathbf{g}_{n+1, p}\right)$. In other words, only the negative terms of $\mathbf{g}_{n+1, p}$, meaning that a penetration has been detected, are kept in $\mathbf{g}_{n+1, p}^{-}$in order to calculate the corresponding corrected displacements $\mathbf{u}_{n+1, c}$.

3. Correction of the displacements through the calculation of normal contact and friction forces (due to high relative velocities between the casing and the blade-tips, it is assumed that only sliding occurs). It yields the addition of the unknown Lagrange multipliers $\boldsymbol{\lambda}$ (or identically $t_{N}$ when using this method) in the governing equations such as

$$
\begin{aligned}
\mathbf{u}_{n+1} & =\mathbf{u}_{n+1, p}+\mathbf{u}_{n+1, c} \\
& =\mathbf{u}_{n+1, p}-\left[\frac{\mathbf{M}}{h^{2}}+\frac{\mathbf{D}}{2 h}\right]^{-1} \mathbf{C}_{\mathbf{N T}} \boldsymbol{\lambda} .
\end{aligned}
$$

The new matrix $\mathbf{C}_{\mathbf{N T}}$ contains the normal and the pure sliding friction constraints. Using the integral form of the contact forces Virtual Work (25) and condition $(22)_{3}$, calculating the terms of the matrix is straightforward. Ultimately, Eqs. (29) and (30) can be recast in such a form that the Lagrange multipliers are solution of

$$
\boldsymbol{\lambda}=\left(\mathbf{C}_{\mathbf{N}}^{T}\left[\frac{\mathbf{M}}{h^{2}}+\frac{\mathbf{D}}{2 h}\right]^{-1} \mathbf{C}_{\mathbf{N T}}\right)^{-1} \mathbf{g}_{n+1, p}^{-} .
$$

\subsection{Contact treatment for modal interaction}

Accurately computing the gap distances between the bodies is of utmost importance. The above algorithm does not give any details about the adopted strategy for the contact detection and the way Eq. (20) is solved. In order to make the description of our approach easier, Fig. 7 specifies all the notations used for the construction of matrices $\mathbf{C}_{\mathbf{N}}$ and $\mathbf{C}_{\mathbf{N T}}$. The master surface is considered to belong to the casing. At each time-step, the absolute angular positions $\beta_{\mid j=1, N}^{j}$ of each blade-tip in the polar coordinate system $\left(e_{r_{c}}, e_{\theta_{c}}\right)$ is computed

$$
\beta^{j}=\Omega t_{n}+\theta_{b}^{j}+\frac{v_{b}^{j}}{R_{b}}, \quad j=1, \ldots, N,
$$

using the following first order expansion

$$
\arctan \left(\frac{v_{b}^{j}}{R_{b}}\right)=\frac{v_{b}^{j}}{R_{b}}+\mathcal{O}\left(\left(\frac{v_{b}^{j}}{R_{b}}\right)^{3}\right) .
$$

Subsequently, a preliminary and straightforward pairing procedure establishes the set $I=$ $\left\{i, \forall j \in[1, N], \beta_{j} \subset\left[\theta_{c}^{i}, \theta_{c}^{i+1}\right]\right\}$ that collects the $N$ elements of the casing possibly impacted by the $N$ blade-tips. In essence, it establishes an implicit relationship between $i$ and $j$. 


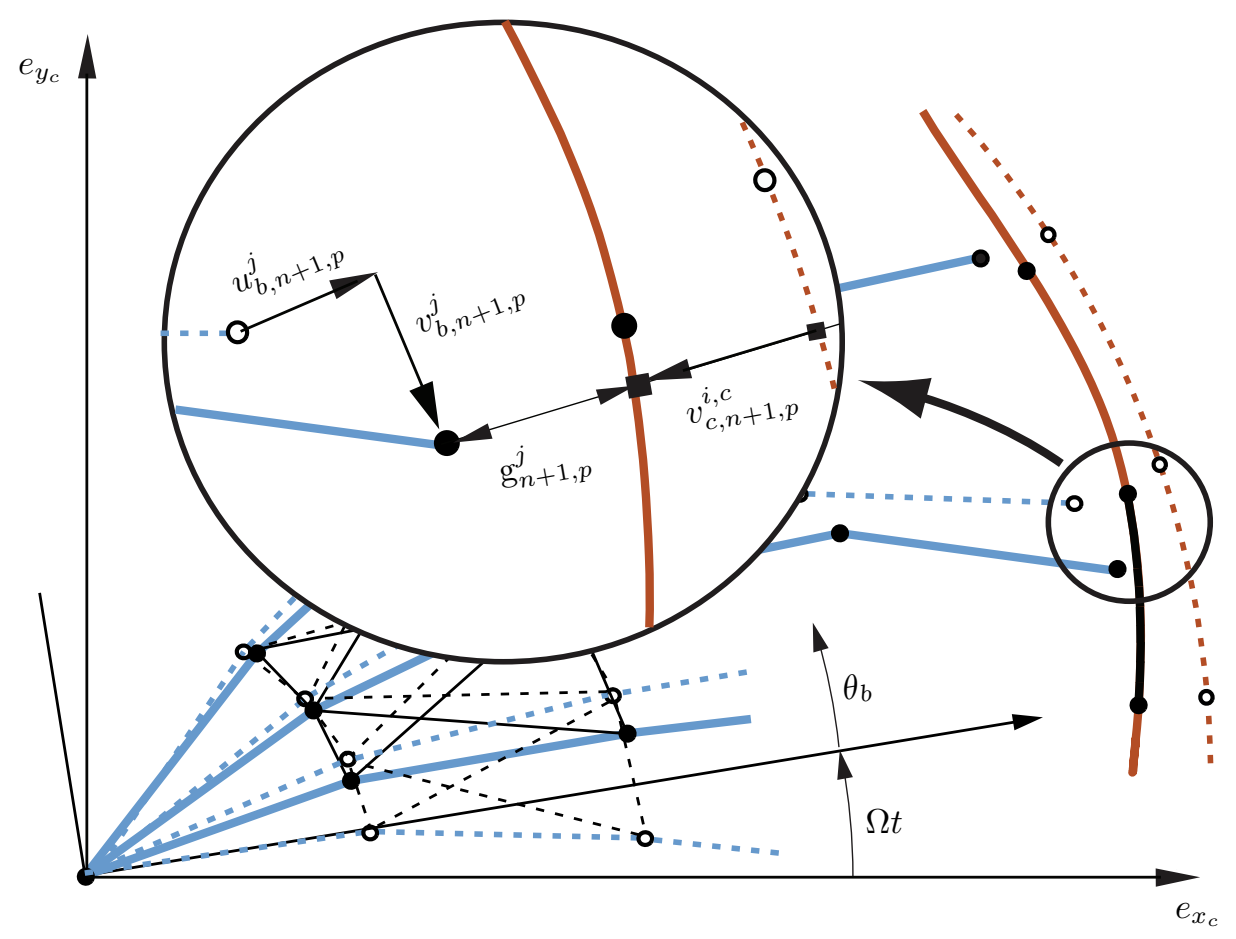

Figure 7: Initial (-..-) and current predicted (-) configurations of a part of the bladed disk and casing

\subsubsection{Contact in the normal direction}

Since all initial angular positions of the nodes of the casing are known in advance, the deduction of $s^{i} \in[0,1]$, the curvilinear path where the tip of blade $j$ is exactly located is of the form

$$
s^{i}=\frac{R_{c}}{l_{c}}\left(\beta^{j}-i\left(\theta_{c}^{i+1}-\theta_{c}^{i}\right)\right), \quad j=1, \ldots, N .
$$

The contact matrix $\mathbf{C}_{\mathbf{N}}$ is then built by writing the distances between the two components. First, these distances depend on the nodal displacements and the shape functions of the casing as depicted in Fig. 7

$$
v_{c, n+1, p}^{i, c}=v_{c, n+1, p}^{i}\left(s^{i}\right), \quad i \in I .
$$

They also depend on the displacements of the blade-tips $u_{b, \mid j=1, N}^{j}$. Reading Eq. (35), it is possible to process, during the prediction step, the gap distance that separates blade $j$ from the facing cross-section $s^{i}$ of the $i^{\text {th }}$ finite element of the casing possibly in contact

$$
\mathrm{g}_{n+1, p}^{j}=R_{c}+v_{c, n+1, p}^{i, c}-R_{b}-u_{b, n+1, p}^{j}, \quad j=1, \ldots, N,
$$

and the expression of the final gap functions with respect to the corrected displacements

$$
\mathrm{g}_{n+1}^{j}=\mathrm{g}_{n+1, p}^{j}+v_{c, n+1, c}^{i, c}-u_{b, n+1, c}^{j}, \quad j=1, \ldots, N .
$$

When generalized to the entire bladed disk, conditions (37) can be gathered in a matrix form similar to Eq. (28)

$$
\mathbf{g}_{n+1}=\mathbf{C}_{\mathbf{N}}^{T} \mathbf{u}_{n+1, c}+\mathbf{g}_{n+1, p} .
$$




\subsubsection{Friction forces}

In normal operating conditions of an aircraft engine, the relative tangential velocity is high enough to ensure that only sliding contact occurs. Accordingly, it is not required to test the location of the contact forces with respect to the Coulomb cone. Then, the friction treatment reduces to adding the appropriate forces during the correction step. It yields the following form for matrices $\mathbf{C}_{\mathbf{N}}$ and $\mathbf{C}_{\mathbf{N T}}$, respectively:

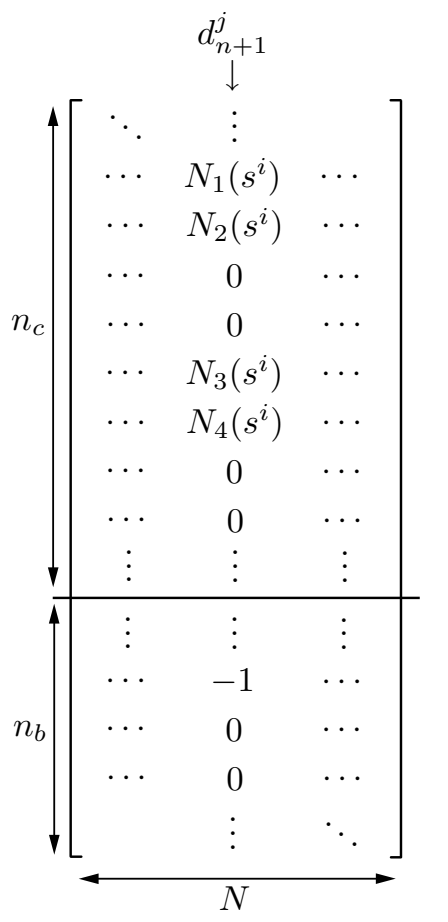

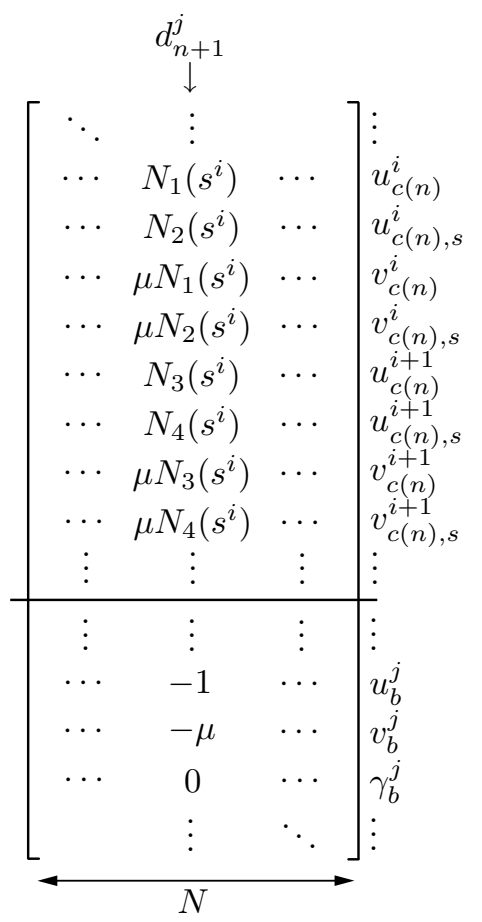

\subsection{Final algorithm}

The method described above needs a minor modification to handle the modal interaction phenomenon. Indeed, the contact constraints can not be treated directly in the chosen modal coordinates of Eq. (17). The final algorithm has to include an alternating procedure between modal and physical coordinates through Eq. (18). The displacements of the structures are predicted in the modal coordinates, projected on the physical coordinates for the contact treatment plus the correction step and finally transformed back to the modal coordinates for the next time step. Therefore, the general form of the algorithm is as follows:

\section{Input:}

- mechanical characteristics of the casing and bladed disk

$-h, k, \Omega$

- initial conditions

- external loads

for $t=t_{i}$ to $t_{f}$ do

- prediction of the displacements $\mathbf{u}_{\mathbf{m}}$ using the modal counterpart of Eq. (27)

- displacement projection onto the physical coordinates using Eq. (18)

- blade-tips to casing gap distances computation using Eq. (36)

if penetration detected then

- Lagrange multipliers computation

- calculation of friction efforts 
- physical displacements correction

end if

- corrected physical displacements projection onto the modal coordinates

- time update

end for

Output:

- modal displacements of the casing $u_{m, c}^{1}$ and $u_{m, c}^{2}$

- modal displacement of the bladed disk $u_{m, b}^{1}$ and $u_{m, b}^{2}$

\section{Modal interaction results}

Tab. 1 sums up the mechanical parameters of our model, adopted in such a way that the eigenfrequencies of the casing are greater than those of the bladed disk in agreement with Fig. 3.

Table 1: characteristics of the model

\begin{tabular}{lll}
\hline & casing & bladed disk \\
\hline Y. modulus $(\mathrm{MPa})$ & $E_{c}=7 \cdot 10^{7}$ & $E_{b}=2.1 \cdot 10^{11}$ \\
density $\left(\mathrm{kg} \cdot \mathrm{m}^{-3}\right)$ & $\rho_{c}=2800$ & $\rho_{b}=7800$ \\
thickness $(\mathrm{m})$ & $h_{c}=0.01$ & $h_{b}=0.01$ \\
width $(\mathrm{m})$ & $w_{c}=0.1$ & $w_{b}=0.1$ \\
radius $(\mathrm{m})$ & $R_{c}=0.4895$ & $R_{b}=0.4795$ \\
d.o.f. & $n_{c}=160$ & $n_{b}=660$ \\
modal damping & $\xi_{c}=0.03$ & $\xi_{b}=0.005$ \\
number of blades & & $N=22$ \\
\hline
\end{tabular}

The contact between the bladed disk and the casing is initiated by a $100 \mu$ s constant forcing pulse on $u_{m, c}^{1}$ at the very beginning of the simulation. During the rest of the simulation no external load is applied. The only remaining forces are the contact and friction forces, if any.

A study of the modal interaction has been performed. The response of the two structures is analyzed with respect to the rotational velocity of the engine and the number of possible simultaneous contact points between the two bodies. It is shown that the type of interaction detected in the simulation can be divided into two main categories depending on the symmetry of the contact locations as depicted in Fig. 8:

1. $N$ is a multiple of $k$ : the distances (and consequently the contact locations) are equally distributed around the perimeter of the casing with respect to $k$, meaning that the number of blade-tips located at the same distance from the casing equals the chosen number of nodal diameters. This specific situation is depicted in Fig. 8(a) where the number of distinguishable curves times the number of nodal diameters $k$ equals the number of blades $N$.

2. $N$ is not a multiple of $k$ : as shown in Fig. 8(b), the blade-to-casing distances do not generally display an axi-symmetric pattern in the circumferential direction. Nevertheless, in very specific cases like the one depicted in Fig. 8(c), for which a symmetric pattern is detected, the gap distances are regularly distributed around the circumference but the 


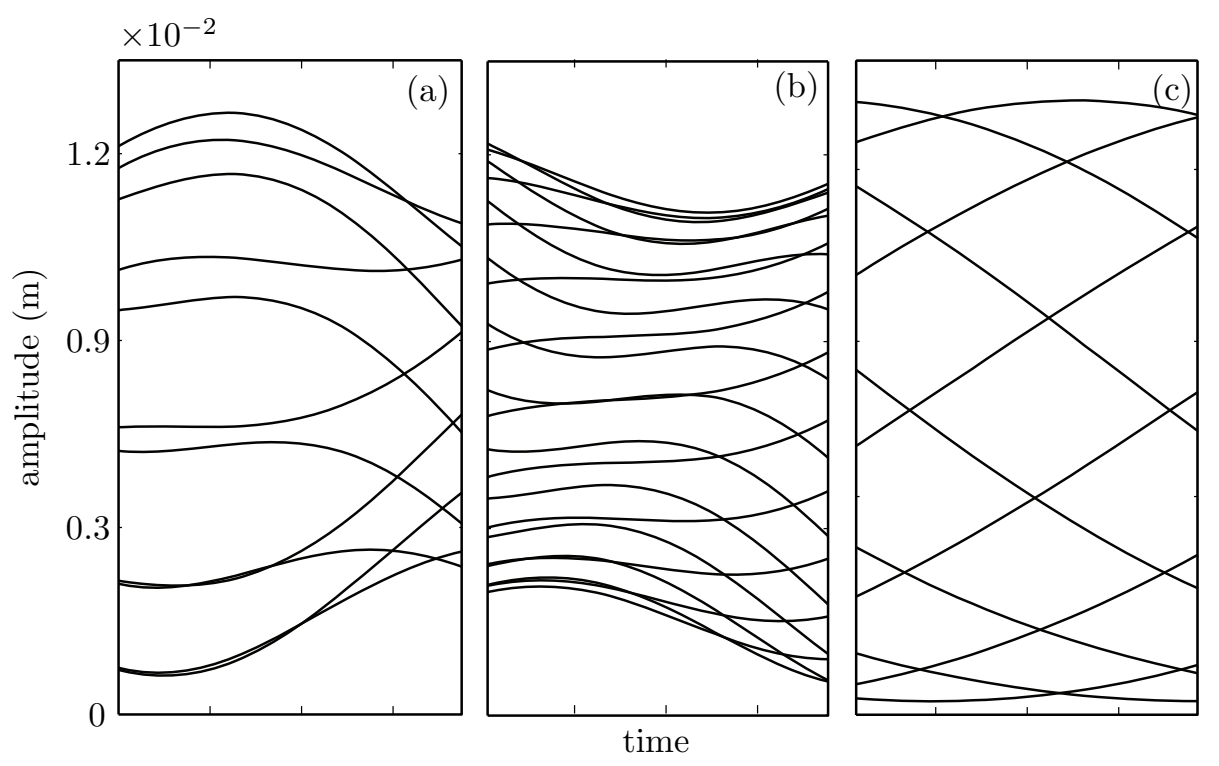

Figure 8: Distances between the casing and the $N=22$ blade-tips before contact is initiated. The time scale is not of interest. A forward $k$-nodal diameter mode is rotating on the casing and a counter-rotating one on the bladed disk. (a) $k=2,11$ curves are distinguishable; (b) $k=3,22$ curves are distinguishable; (c) $k=4,11$ curves are distinguishable. The number of distinguishable curves shows the symmetry of the system in terms of future contact locations between the two components versus $N$ and $k$.

number of blades possibly in simultaneous contact with the casing is different from the number of nodal diameters $(22 / 11=2$ versus $k=4)$.

As a matter of fact, the difference in behavior versus the ratio $N / k$ resides in the axi-symmetric distribution of the blades possibly and simultaneously in contact with the outer casing. When the chosen geometry allows for the system to be perfectly symmetric in terms of $N$ and $k$, the contact points will remain at the same blade tips for all times. In the rotating frame rigidly linked to the rotor, the contact and friction forces can be considered as constant, then explaining the static deformed shape reached by the structure. On the other hand, when $N / k$ is not an integer, the contact positions will move among the blade-tips and excite, due to friction and intermittent contact, a counter-rotating mode in the bladed disk, as well as, in agreement with Newton's third law, a forward rotating mode in the casing. This observation is reinforced by several numerical simulations involving different parameter pairs $(N, k)$ like $(22,2),(22,3)$, $(22,4)$ or $(24,3),(24,4)$ and $(24,5)$ for instance. This behavior is not predicted by the analytical statements.

\section{$5.1 N / k$ is not an integer $(N=22, k=4)$}

Under these assumptions, the coupling by means of structural contacts between the two components of the aircraft engine model exhibits three different types of response in terms of $\Omega$; the boundaries of the different system behaviors are defined with respect to a critical velocity denoted $\Omega_{c n}$, numerical counterpart of the theoretical critical velocity $\Omega_{c t}$, solution of Eq.(11):

1. $\boldsymbol{\Omega}<\boldsymbol{\Omega}_{\boldsymbol{c n}}$ : the response is characterized by several impacts between the two structures at the beginning of the simulation due to the initial pulse on the casing. They are followed by 


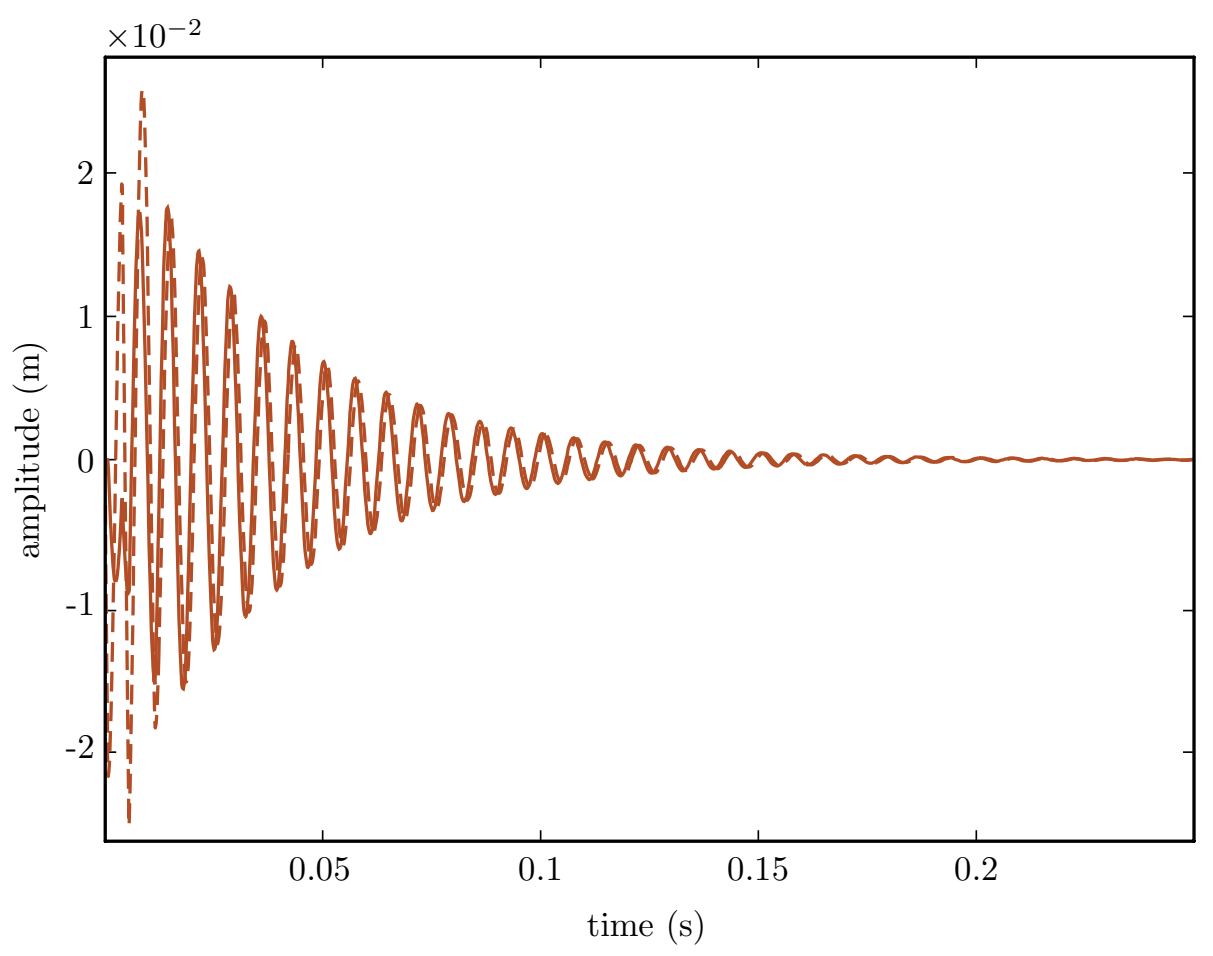

Figure 9: Vibrations of the two modes of the casing for $\Omega=350.2 \mathrm{rad} / \mathrm{s}\left(\Omega<\Omega_{c n}\right)$ : $u_{m, c}^{1}(---)$ and $u_{m, c}^{2}(-)$. A decaying behavior of the casing is observed due to the presence of structural damping in the model.

a decrease of the amplitudes of vibration to zero stemming from the structural damping as shown in Fig. 9. This case is called "damped motion".

2. $\boldsymbol{\Omega} \simeq \boldsymbol{\Omega}_{\boldsymbol{c} \boldsymbol{n}}$ : the modal shapes of both structures geometrically match each other and structural contacts with friction allow for a forward rotating mode in the casing and a backward one in the bladed disk to occur. Both structures vibrate close to their own natural frequency yielding an aperiodic (made of two incommensurable frequencies $\omega_{c}$ and $\left.\omega_{b d}\right)$ limit cycle. The phase shift between the two curves in Fig. 11 plotting the time evolution of the two modes of the casing is an illustration of a wave propagation whose mathematical expression is given by Eq. (8). This case is called "sustained motion".

3. $\boldsymbol{\Omega}>\boldsymbol{\Omega}_{\boldsymbol{c n}}$ : the behavior of the system is unstable as shown in Fig. 13 for the casing and Fig. 14 for the bladed disk. The vibration amplitudes become extremely large after about a hundred of rotor rounds. This case is called "divergent motion".

Naturally, the vibrations of the bladed disk are consistent with those of the casing. They meet exactly the same classification: (1) a decaying behavior (see Fig. 10), (2) a sustained motion (see Fig. 12) and (3) a divergent behavior (see Fig. 14).

Critical velocities $\Omega_{c t}$ and $\Omega_{c n}$ can be compared for $k=4$,

$$
\Omega_{c t}=343.58 \mathrm{rad} / \mathrm{s} \text { and } \Omega_{c n}=351.4 \mathrm{rad} / \mathrm{s} \text {. }
$$

Calculations for $k=3$ and $k=5$ have been carried out in order to ensure that the developed algorithm was predictive. Respective results are given in Fig. 15. This figure shows good agreement between the analytical approach and the numerical tool even though analytical and numerical values of the critical rotational velocity are not exactly identical. Moreover, the 


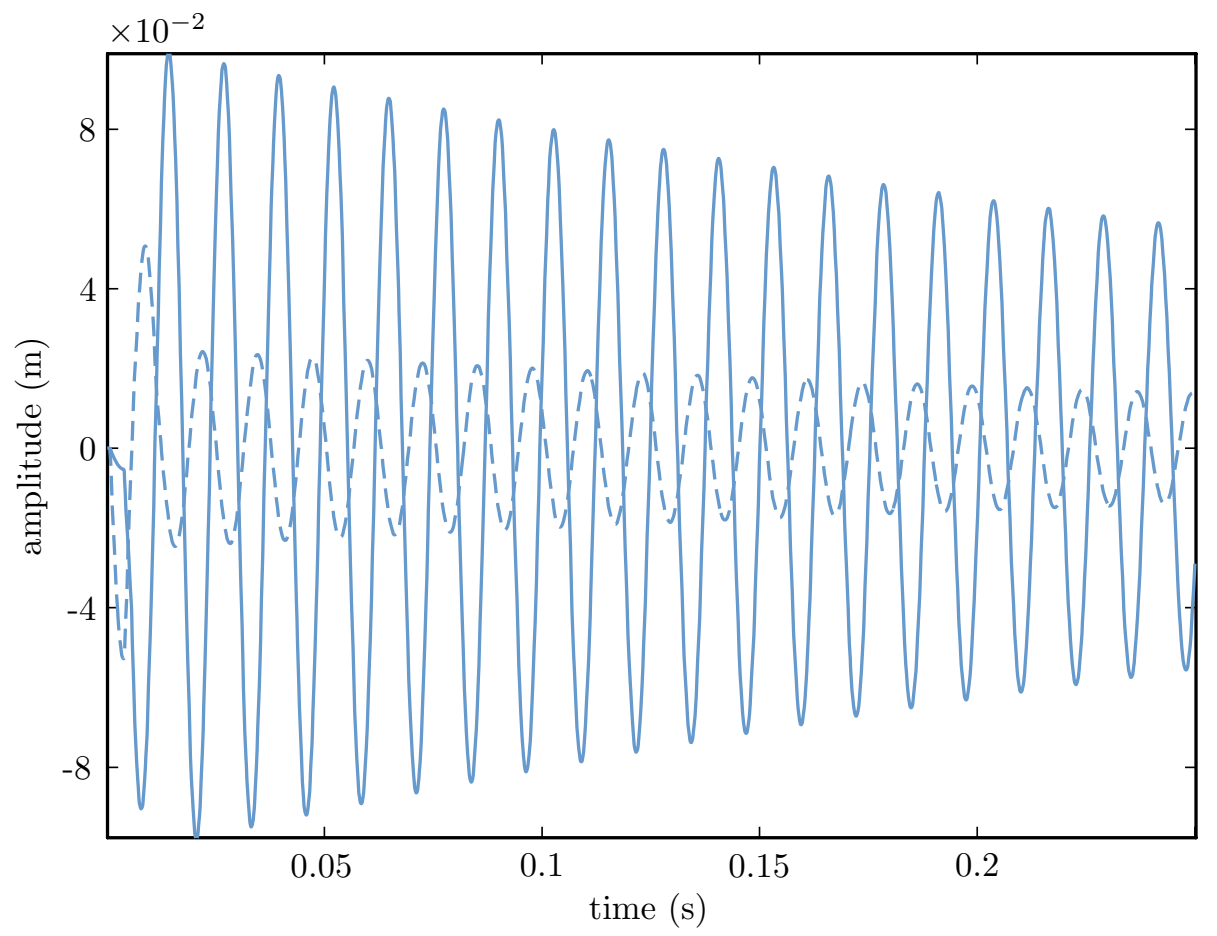

Figure 10: Vibrations of the two modes of the bladed disk for $\Omega=350.2 \mathrm{rad} / \mathrm{s}\left(\Omega<\Omega_{c n}\right)$ : $u_{m, b}^{1}(---)$ and $u_{m, b}^{2}(-)$. A decaying behavior of the bladed disk is observed due to the presence of structural damping in the model.

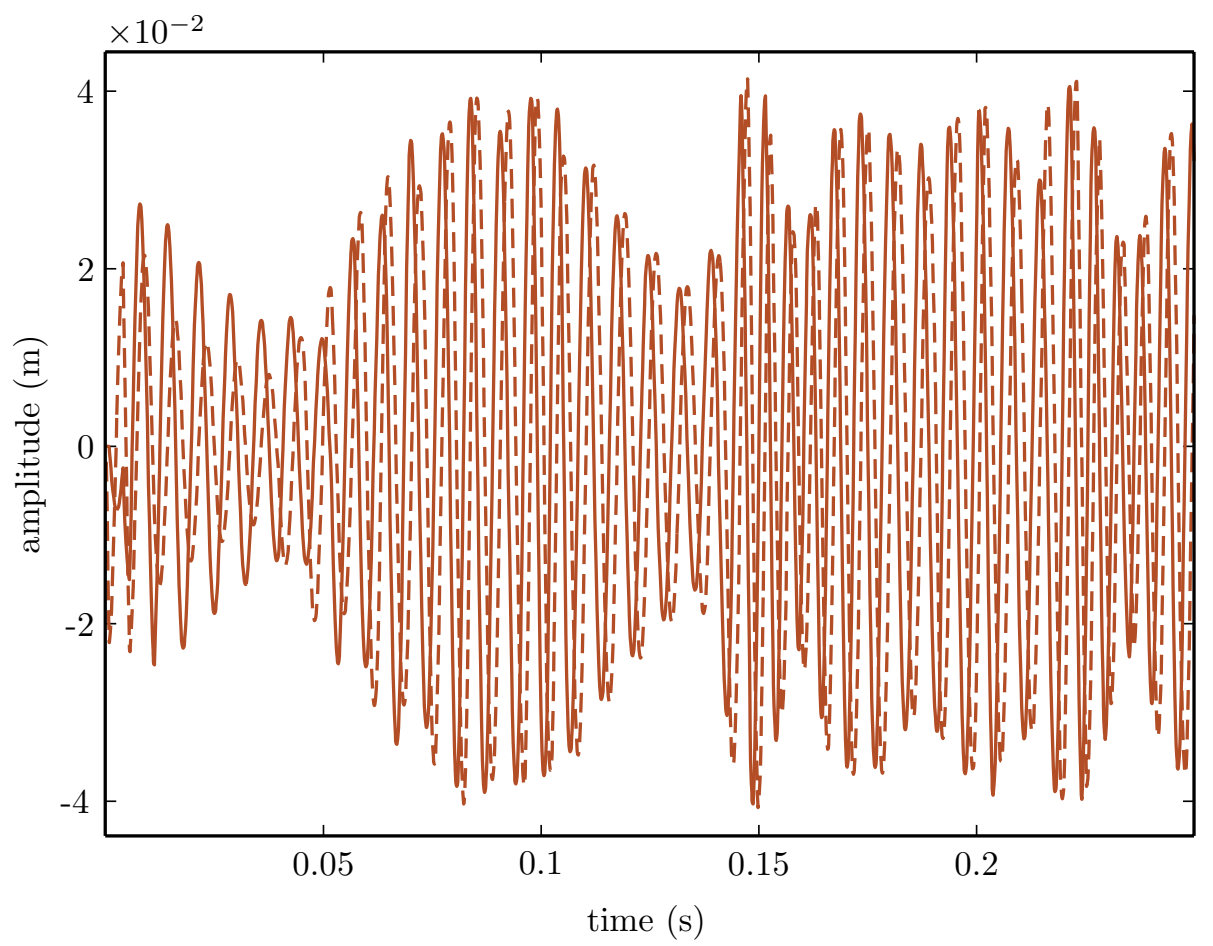

Figure 11: Vibrations of the two modes of the casing for $\Omega=351.4 \mathrm{rad} / \mathrm{s}\left(\Omega \simeq \Omega_{c n}\right)$ : $u_{m, c}^{1}(---)$ and $u_{m, c}^{2}(-)$. A forward rotating mode propagates in the casing. 


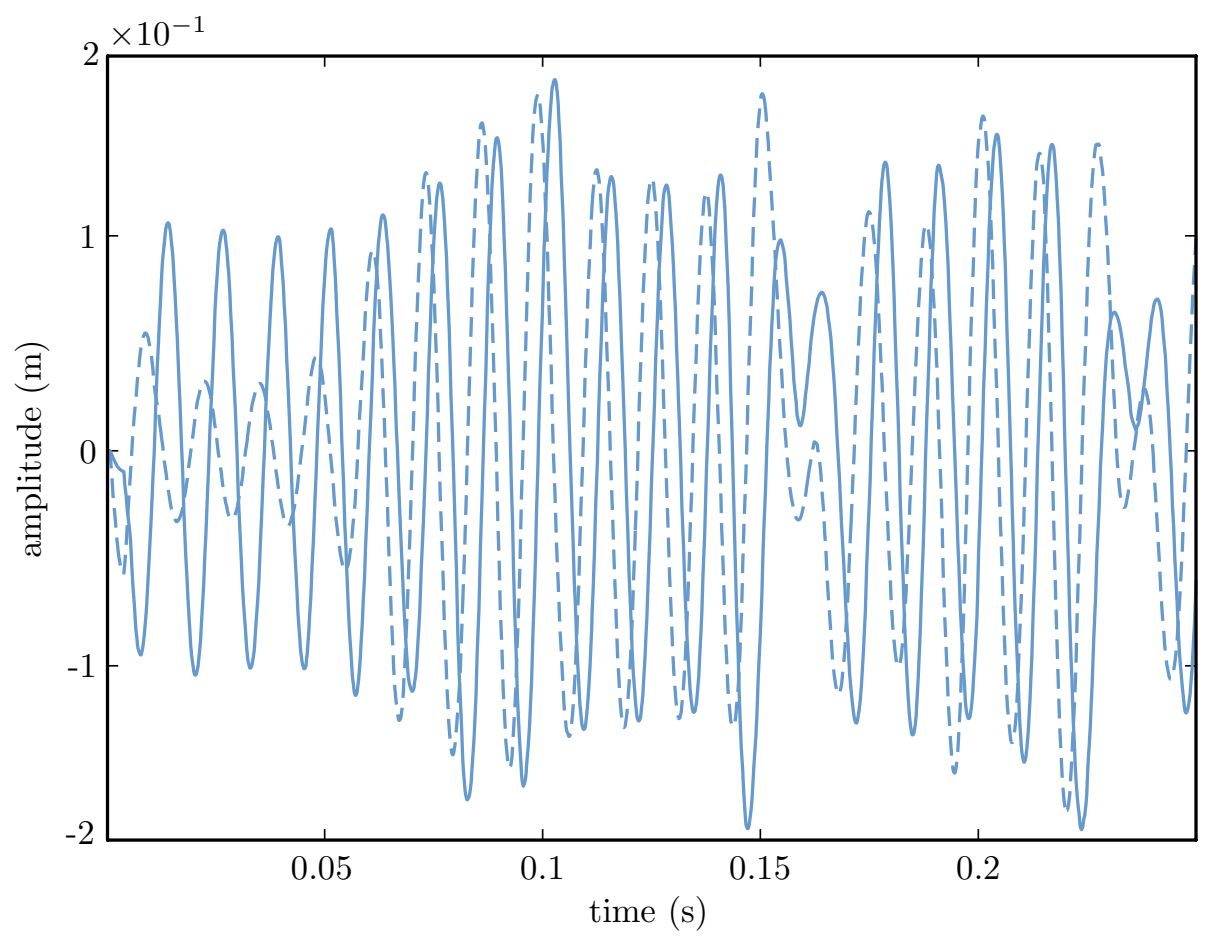

Figure 12: Vibrations of the two modes of the bladed disk for $\Omega=351.4 \mathrm{rad} / \mathrm{s}\left(\Omega \simeq \Omega_{c n}\right)$ : $u_{m, b}^{1}(---)$ and $u_{m, b}^{2}(-)$. A counter-rotating mode propagates in the bladed disk.

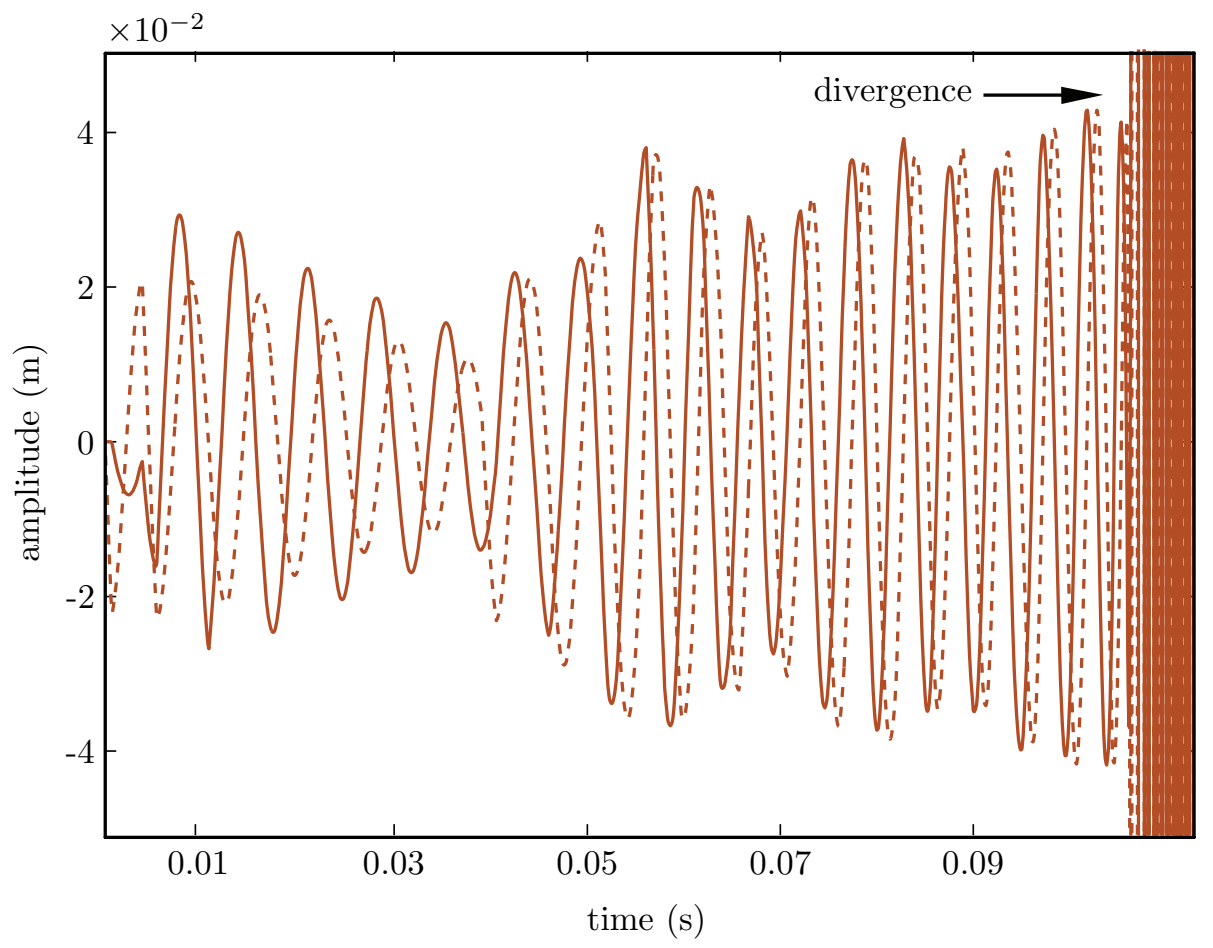

Figure 13: Vibrations of the two modes of the casing for $\Omega=351.8 \mathrm{rad} / \mathrm{s}\left(\Omega>\Omega_{c n}\right)$ : $u_{m, c}^{1}(---)$ and $u_{m, c}^{2}(-)$. The amplitudes of vibration diverge very quickly.

analytical approach predicts only one dangerous theoretical rotational velocity whereas the nu- 


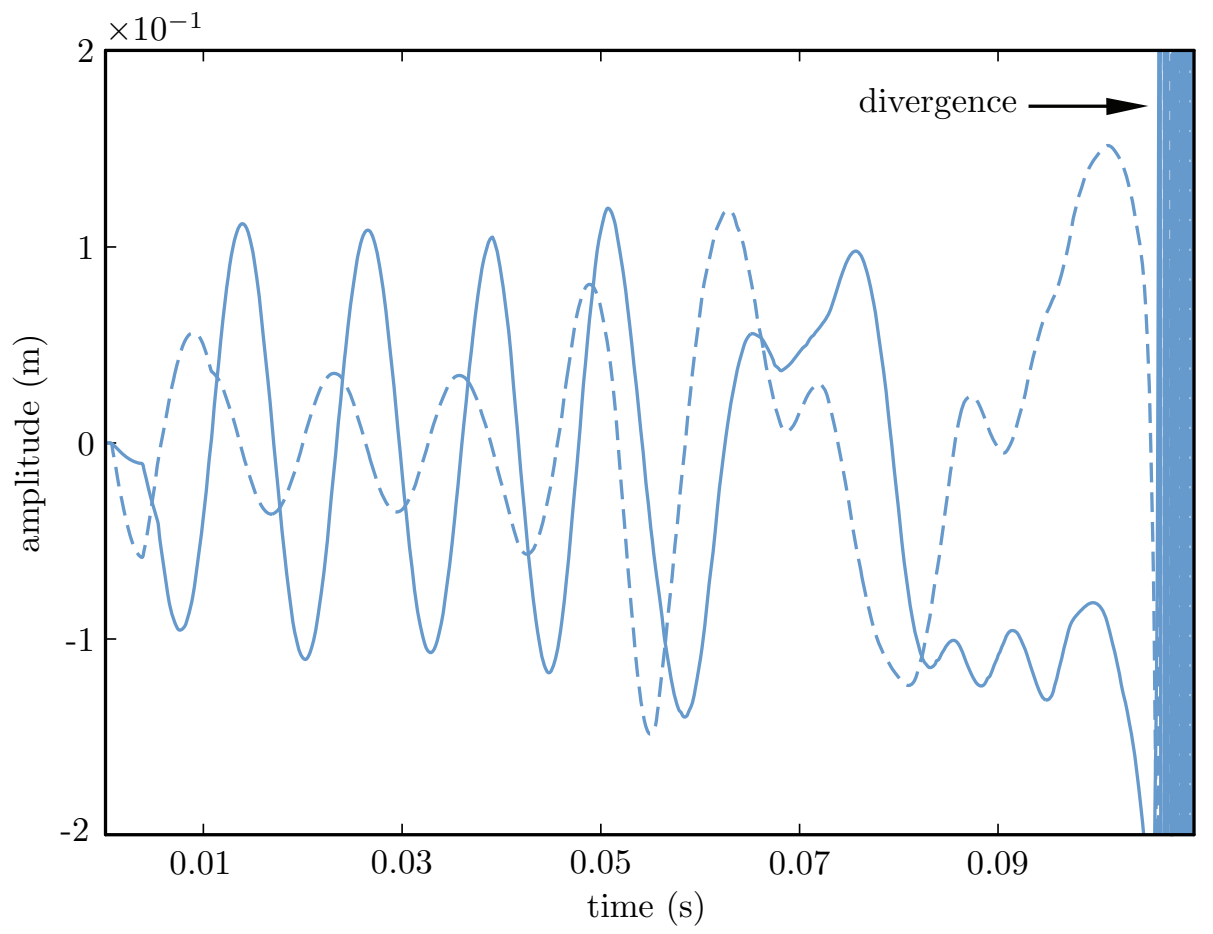

Figure 14: vibrations of the two modes of the bladed disk for $\Omega=351.8 \mathrm{rad} / \mathrm{s}\left(\Omega>\Omega_{c n}\right)$ : $u_{m, b}^{1}(---)$ and $u_{m, b}^{2}(-)$. The amplitudes of vibration diverge very quickly.

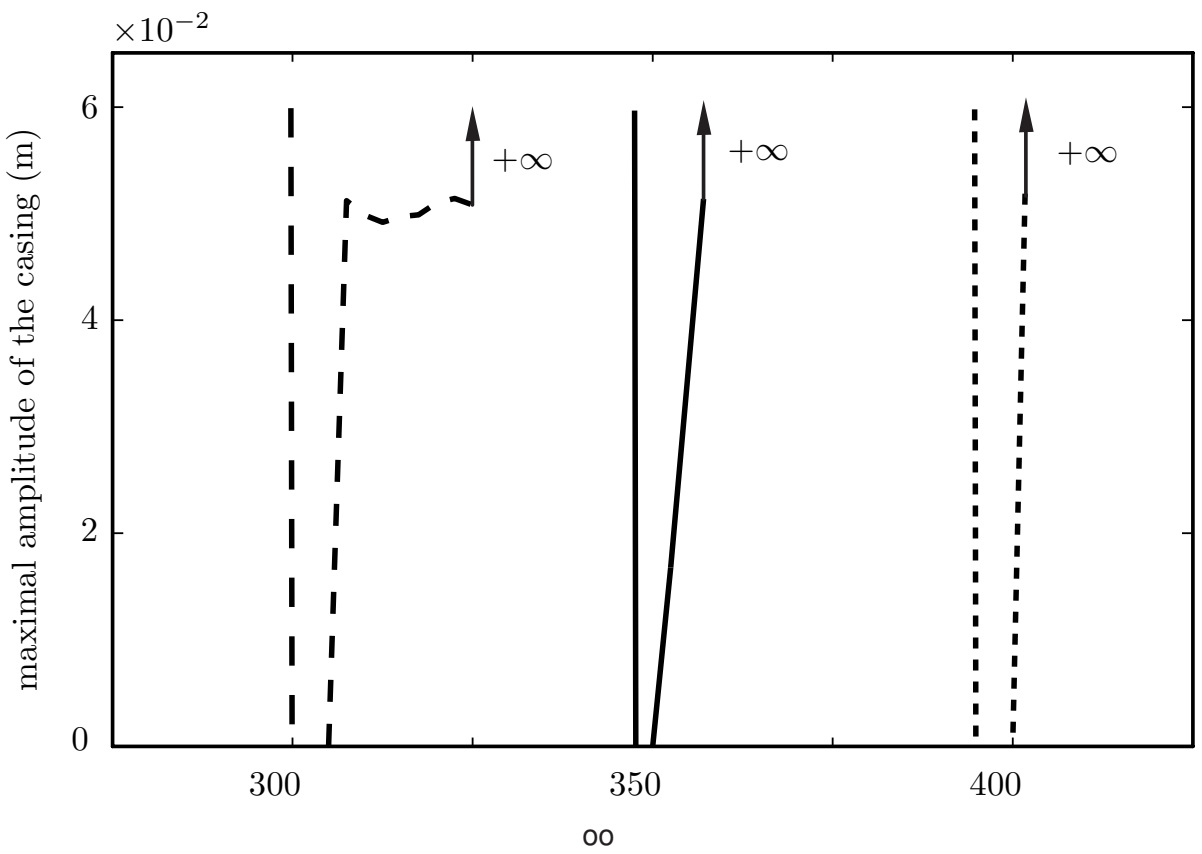

Figure 15: Critical rotational velocities, with on the left, the analytical value and on the right, the numerical value; $(--): k=3, \Omega_{c t}=301.56 ;(-): k=4, \Omega_{c t}=343.58 ;(---): k=5$, $\Omega_{c t}=390.37$.

merical tool predicts a range of rotational velocities for which the interaction becomes unstable, 


$$
\Omega \geq \Omega_{c n} \simeq \Omega_{c t}=\frac{\omega_{c}+\omega_{b d}}{k} .
$$

A very interesting feature of the "sustained motion", which can be seen in Fig. 16, is the intermittent nature of the contact pattern which is endlessly repeated: the blades are touching the casing one after another (with a given number of blades simultaneously in contact with the casing) then, the contact is lost until the pattern starts again. This is conform with the ideal behavior shown in Fig. 4.

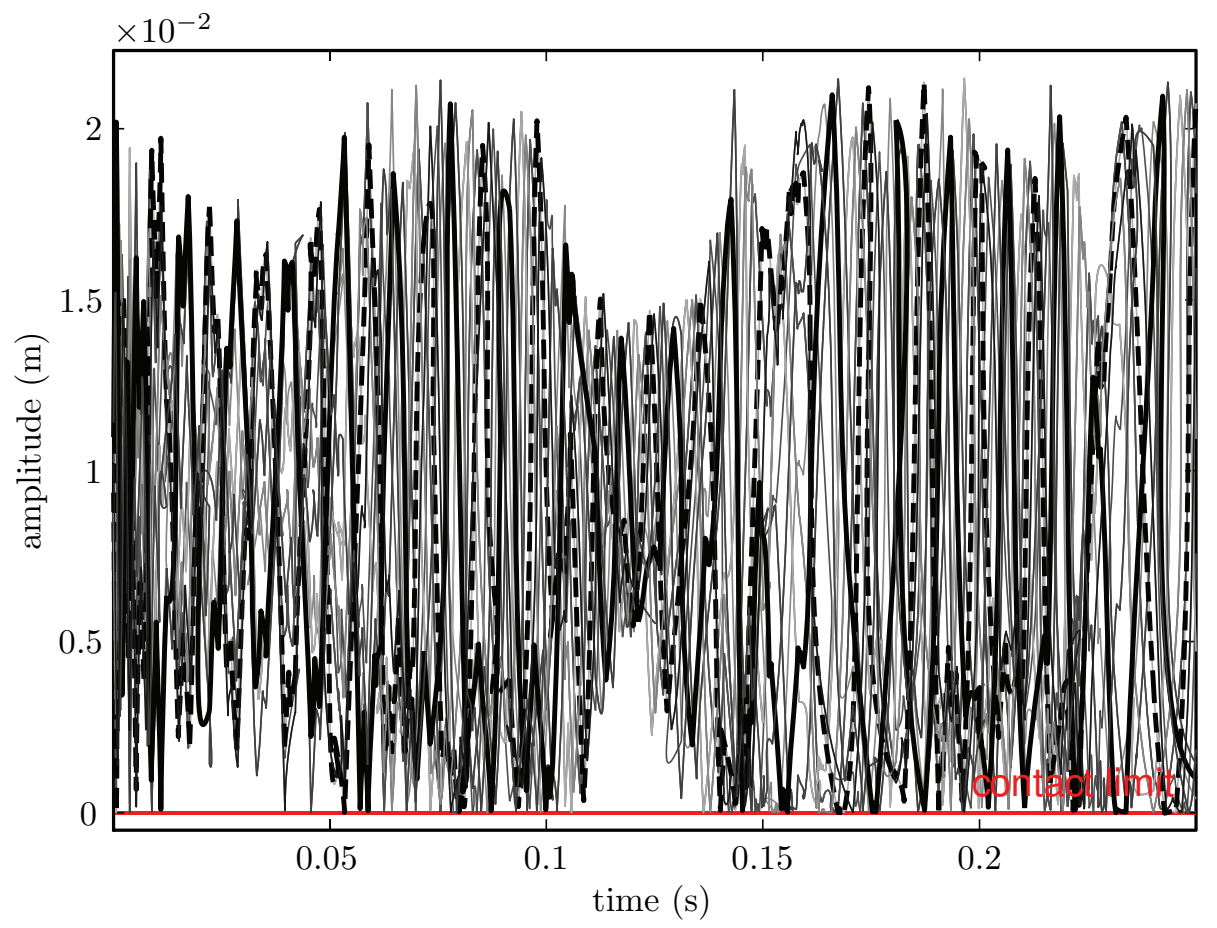

Figure 16: Distances between the casing and blade-tips for $N=22, k=3$ and $\Omega=351.4 \mathrm{rad} / \mathrm{s}$. The intermittent behavior can be observed for two highlighted blades (dash and solid bold black lines).

\section{$5.2 N / k$ is an integer $(N=22, k=2)$}

Under these assumptions, both structures exhibit only two types of response whose description is different from the previous example since the critical angular velocity $\Omega_{c n}$ has to be redefined:

1. $\boldsymbol{\Omega}<\boldsymbol{\Omega}_{\boldsymbol{c} \boldsymbol{n}}$ : the response is characterized by several impacts between the two structures at the beginning of the simulation due to the initial pulse on the casing. They are then followed by a decay of the amplitudes of vibration to zero stemming from the structural damping.

2. $\boldsymbol{\Omega} \geq \boldsymbol{\Omega}_{\boldsymbol{c n}}$ : after a transient response, a static deformed state of the bladed disk pushes a forward rotating mode in the casing (see Fig. 17 and 18). Only a few blades remain in permanent contact with the outer casing as depicted in Fig. 19. During the interaction, the vibrations of the casing are bounded and no divergent behavior has been found.

As the bladed disk no longer vibrates when the interaction phenomenon is reached, the critical rotational velocity is related to the natural frequency of the casing only. Numerically, it 


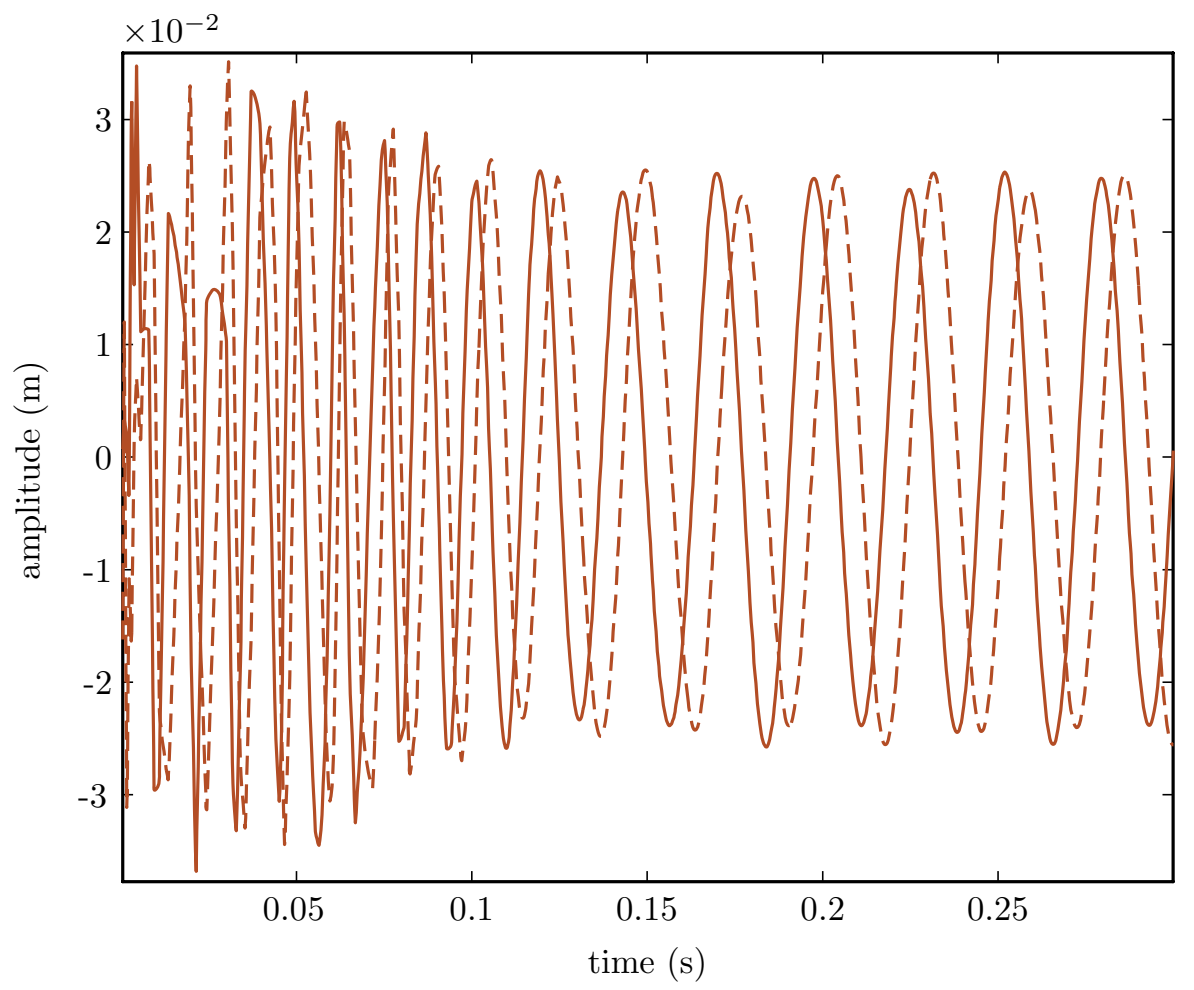

Figure 17: Vibrations of the two modes of the casing for $\Omega=110 \mathrm{rad} / \mathrm{s}\left(\Omega>\Omega_{c n}\right)$ : $u_{m, c}^{1}(---)$ and $u_{m, c}^{2}(-)$. A forward rotating mode starts traveling after $0.14 \mathrm{~s}$ of simulation.

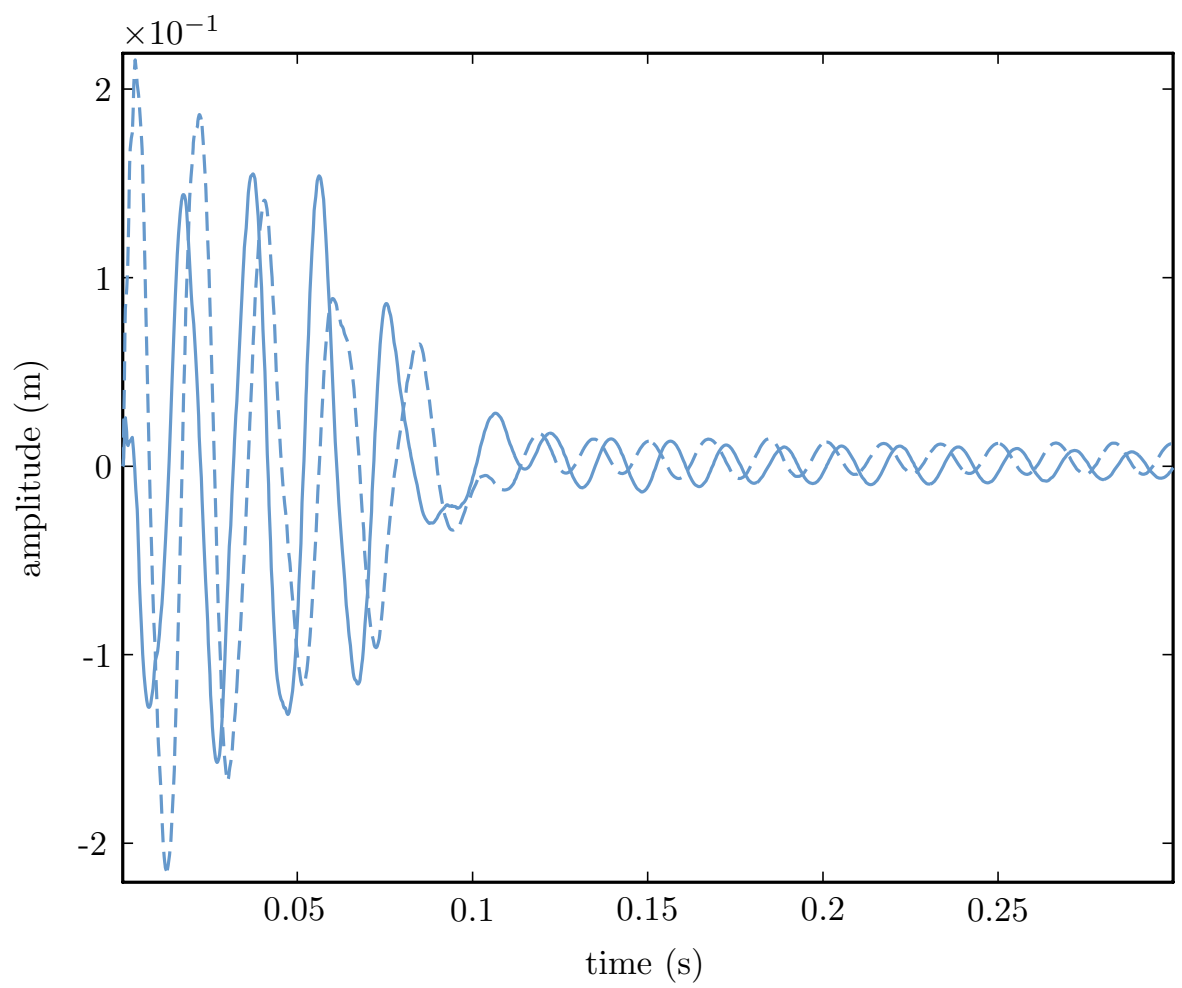

Figure 18: Vibrations of the two modes of the bladed disk for $\Omega=110 \mathrm{rad} / \mathrm{s}\left(\Omega>\Omega_{c}\right)$ : $u_{m, b}^{1}(---)$ and $u_{m, b}^{2}(-)$. They are decaying until a static deformed state is reached. 


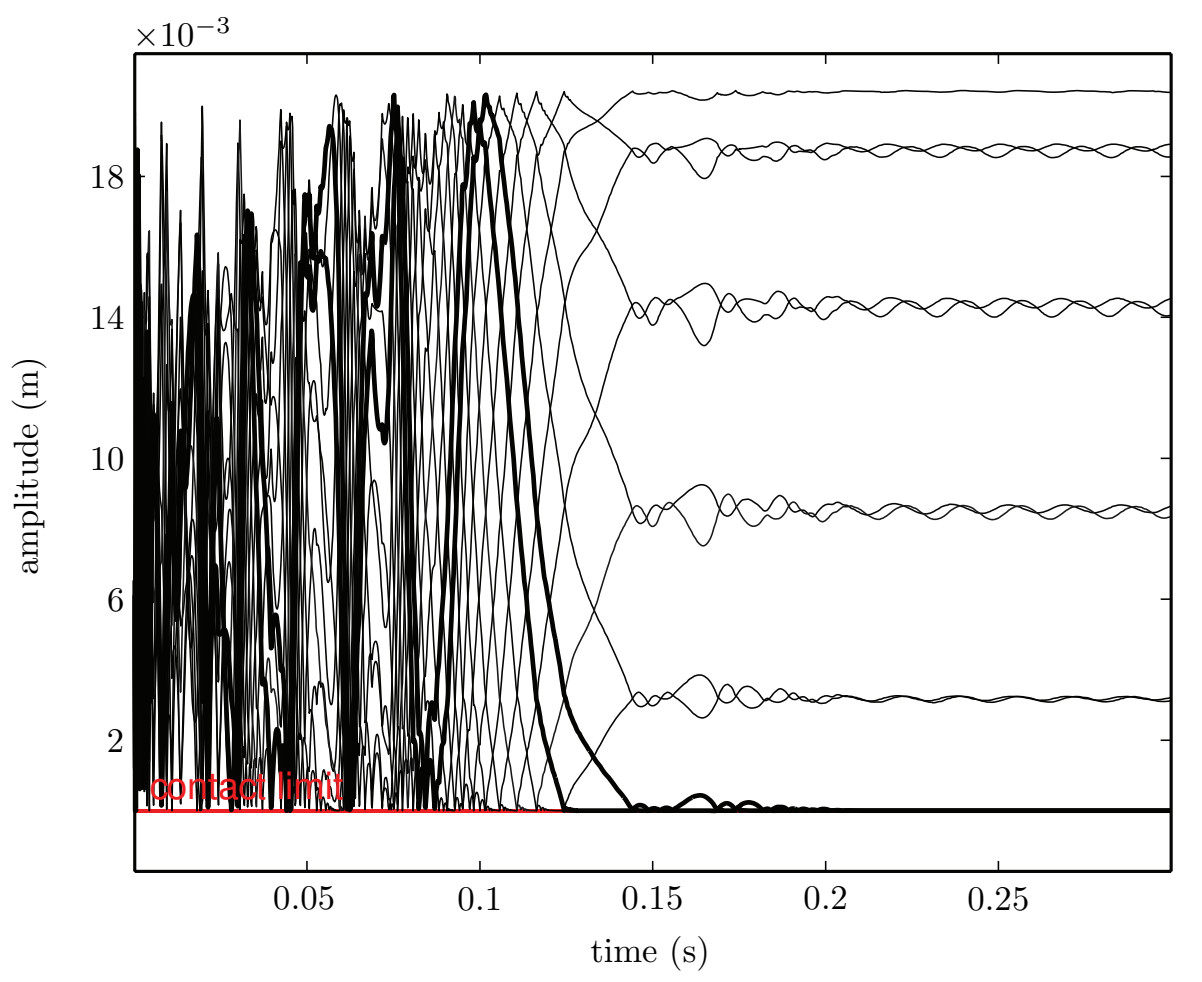

Figure 19: Distances between the casing and the blade tips for $\Omega=110 \mathrm{rad} / \mathrm{s}$. Due to the symmetry of the system, only 11 curves are distinguishable. After $0.14 \mathrm{~s}$ of simulation, four blades ( 4 solid bold black curves are shown but only 2 are distinguishable) come in permanent contact against the casing and push a forward rotating mode.

can be shown that a case of non-divergent interaction with permanent contact is reached only if $\Omega \geq \Omega_{c n}$ where

$$
\Omega_{c n}=\frac{\omega_{c}}{k} .
$$

This series of results highlights the potential of our predicting numerical tool that supplements the presented analytical approach. It is capable of detecting different, rich motions and limit cycles the system may experience as well as handling parameter studies on blade curvature, friction coefficient or structural damping to name a few. As a simple frequency argument, this analytical approach, already introduced in [7,8], clearly cannot predict physical behaviors even though it brings a first very important insight of the phenomenon, the interaction condition.

\section{Further results: parameter study}

Based on the previous "nominal" results, it is obviously of interest for a manufacturer to draw tendencies with respect to a set of mechanical parameters considered as crucial. In what follows, a parameter study has been performed with respect to (1) the reference curvature of the blades and (2) the coefficient of friction $\mu$.

\subsection{Reference blade curvature}

Different curvature configurations shown in Fig. 20, identical for all blades whose reference positions are illustrated in Fig. 5, have been tested. It is aimed at categorizing possible geometries, 


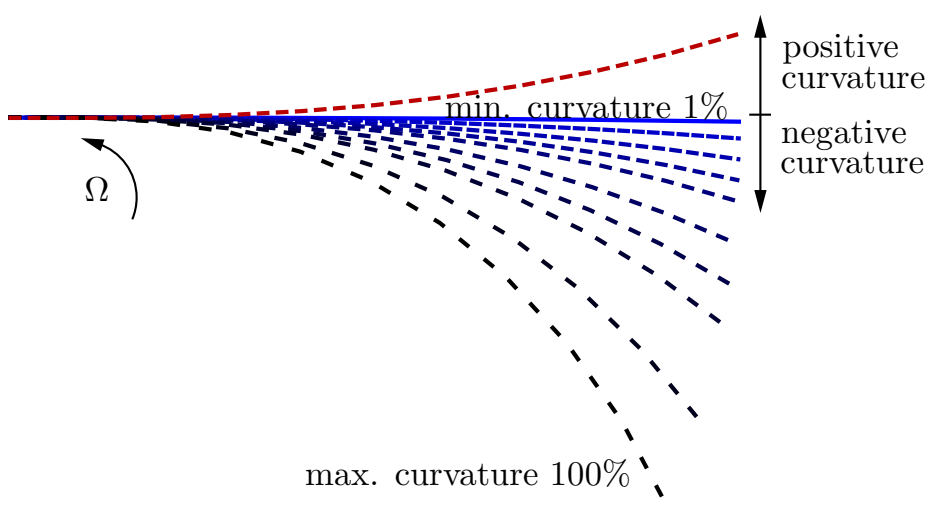

Figure 20: Classification of the tested blade curvatures from 1\% (almost straight) to $100 \%$ (highly curved). A blade with positive curvature is also plotted.

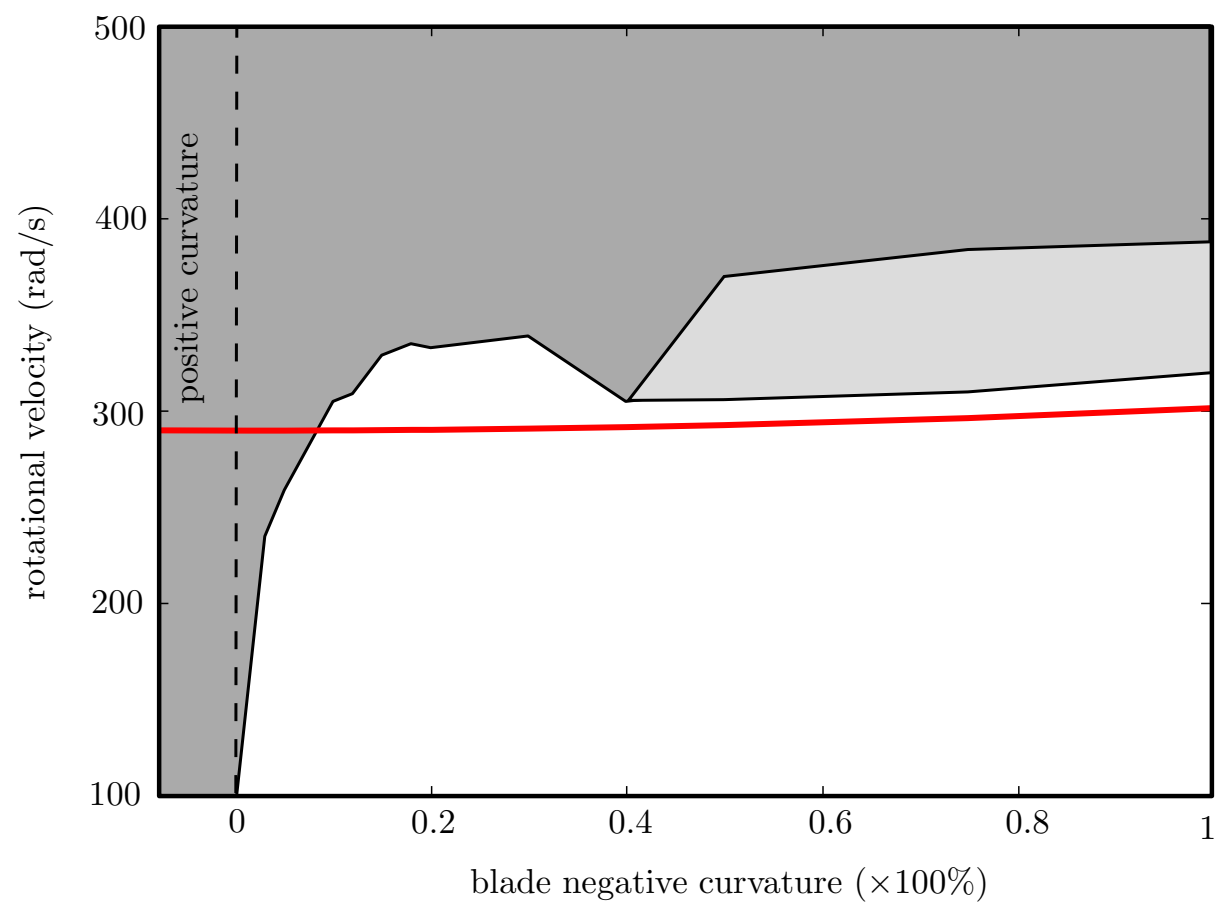

Figure 21: The different possible vibratory behaviors of the structures are given with respect to the blades curvature and the rotational velocity; $N=22, k=3$ and $\mu=1 ; \square$ damped, sustained, $\square$ divergent, and — analytical solution.

favorable or not to the occurrence of the interaction phenomenon. Firstly, a positive curvature has the unsatisfactory effect of closing the tip clearance when the blades are excited by the friction forces and leads to instantaneous divergent behaviors, even for low rotational velocities and very soft initial contacts. Secondly, the classification with negative curvature designs, given in Fig. 21, is more complex. It is worth stating that the original blade design used in the previous sections is considered here to be the $100 \%$ curvature design. Various simulations performed in the "curvature, rotational velocity" plane and thorough examinations of the numerical solutions allow for drawing the boundaries that separate the different possible motions listed above for $N=22$ and $k=3$. It is shown that under a threshold around $40 \%$ of curvature, a sustained motion is impossible. In this zone, a rising $\Omega$ causes a jump directly from a damped motion 
to a divergent behavior, which clearly must be avoided. It is also worth noting that above this threshold, the difference between the theoretical critical speed which naturally evolves with the curvature and the lower boundary of the sustained motion zone remains constant. This tiny difference confirms the predicting performance of the proposed method. Moreover, it may be explained by the presence of structural damping and friction in the model which are not taken into account in the analytical approach.

\subsection{Friction coefficient}

As a first estimation, we can legitimately assume that a larger friction coefficient would reduce the interaction phenomenon by dissipating a larger amount of energy that could transfer from one component to the other. From Fig. 22, it is apparent that in reality, a larger friction coefficient increases the possibility of undesirable motions with large amplitudes. Fundamentally, the sustained motion zone tends to be replaced by a divergent behavior zone with larger friction coefficient. In other words, it indicates that a divergent behavior is more likely to appear with higher friction coefficients. Finally, the (partial) agreement between the two presented approaches, underlines the potential threat of the phenomenon of interest. It has to be considered with attention by aircraft engine manufacturers for flights safety. A safe reduction of

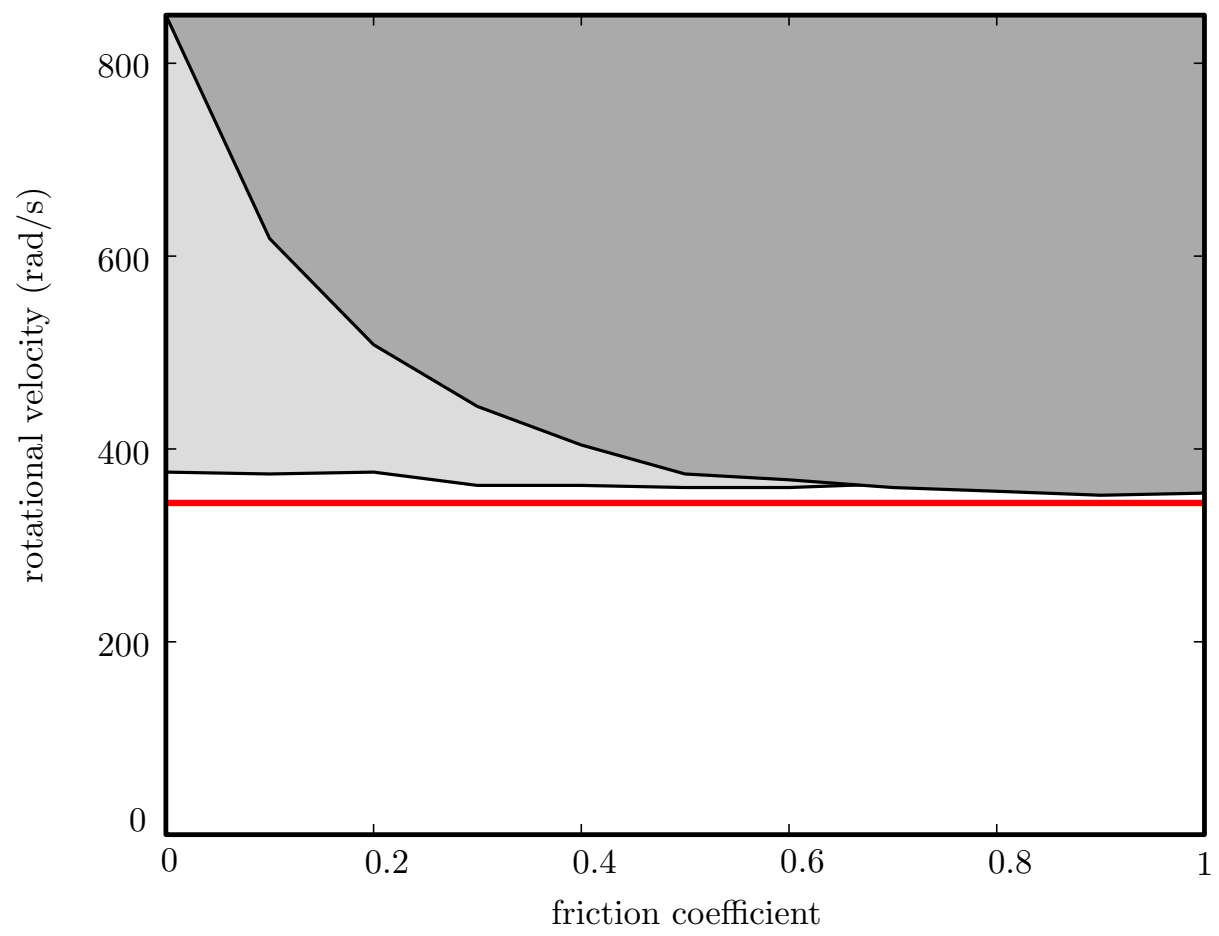

Figure 22: The different possible vibratory behaviors of the structures are given with respect to the friction coefficient and the rotational velocity; $N=22$ and $k=4$. The blades curvature is $70 \%$; $\square$ damped, $\square$ sustained, $\square$ divergent, and — analytical solution.

the tip clearance is usually obtained by using an abradable coating laid on the casing [26]. The modeling of such a material requires a wear law and represents a difficult task. Even though it is beyond the scope of the present paper, it has to be included in our numerical tool in a future work. 


\section{Conclusions}

The emphasis of the study has been placed on the understanding of the modal interaction caused by direct contact that may occur between a bladed disk and a casing in an aircraft engine due to the small tip clearance.

A very simple analytical and linear approach is available for obtaining aircraft engine critical velocities in terms of modal interaction ${ }^{2}$. Unfortunately, by neglecting the contact constraints and structural damping among others, it only furnishes a poor description of the phenomenon. Moreover, it is unable to predict more complex and purely nonlinear behaviors like the interaction between different nodal diameter modes respectively travelling on the casing $(k=3)$ and on the bladed-disk $(k=6)$. Accordingly, a numerical tool is greatly justified.

The advances given in this paper primarily relate to the structural modeling and treatment of the contact conditions used in the proposed numerical tool. In order to capture this phenomenon and get interesting and realistic results, it is necessary (1) to choose a geometric design that couples axial and flexural displacements and (2) to include friction forces in the contact law in a consistent and accurate manner. In our approach, the first condition is achieved by curving the blade geometry and the second one, by an explicit time-stepping procedure in conjunction with the method of Lagrange multipliers where the usual Coulomb's friction law is accounted for.

As far as this study shows, a jet engine may experience different kinds of modal interactions depending on the number and curvature of the blades, the number of nodal diameters of the rotating modes, the friction coefficient. Under certain conditions, it is also shown that the analytical statements are valuable for testing the performances of the proposed algorithm and qualitatively consistent with the numerical method.

Even though the presented 2D numerical tool has proved to be suitable for complex behaviors prediction and quick parameter studies, it is still very limited compared to full 3D investigations. For instance, recent blade designs, using forward sweep for performance and stability purposes [27], resemble the positive curvature geometry introduced in the present 2D numerical tool. Based on the shown results, blade geometries that tend to close the clearance gap with the casing during interaction lead to quick divergence. As a consequence, research attention has to be paid to such designs that better satisfy aerodynamic engineers but may lead to structural failure in case of interaction, depending on the type of mode involved (flexural or torsional). Solutions to reduce the risk of failure that were not explored in the present paper have already been suggested, such as intentional mistuning (intentional amount of disorder in the structures in order to disrupt the existence of nodal diameter rotating modes) [28] or abradable coatings whose modeling remains a very difficult task. Extension of this tool to examine new issues such as acoustically-coupled resonances [29] may also be considered. Undoubtedly, avenues for future research work are numerous and should be complemented by thorough experimental studies. Ultimately, the purpose of such a numerical tool is to be part of a broader design process aimed at optimizing the structures in presence and reducing the occurrence of this phenomenon. It may also be important to identify the origin(s) of the differences between the present investigation and [7], especially in terms of rotational velocities for which a divergence is detected.

\section{Acknowledgement}

Thanks go to Snecma for its technical and financial support. This work takes place in the framework of the MAIA mechanical research and technology program sponsored by CNRS,

\footnotetext{
${ }^{2}$ It is understood here that other "critical velocities", due to flutter for instance, are not of interest in the present work.
} 


\section{A Appendix. Vocabulary}

\section{Traveling waves}

Some terms specific to flexible structures and rotordynamics may be confusing. Below, we want to clarify the four following directional terms associated with waves traveling on structures that exhibit axi-symmetry (including rotationally periodic structures): forward, backward, corotating and counter-rotating. For the sake of simplicity, the counter-clockwise direction is selected as positive. By definition, a forward wave propagates at speed $\nu_{f}$ in the positive

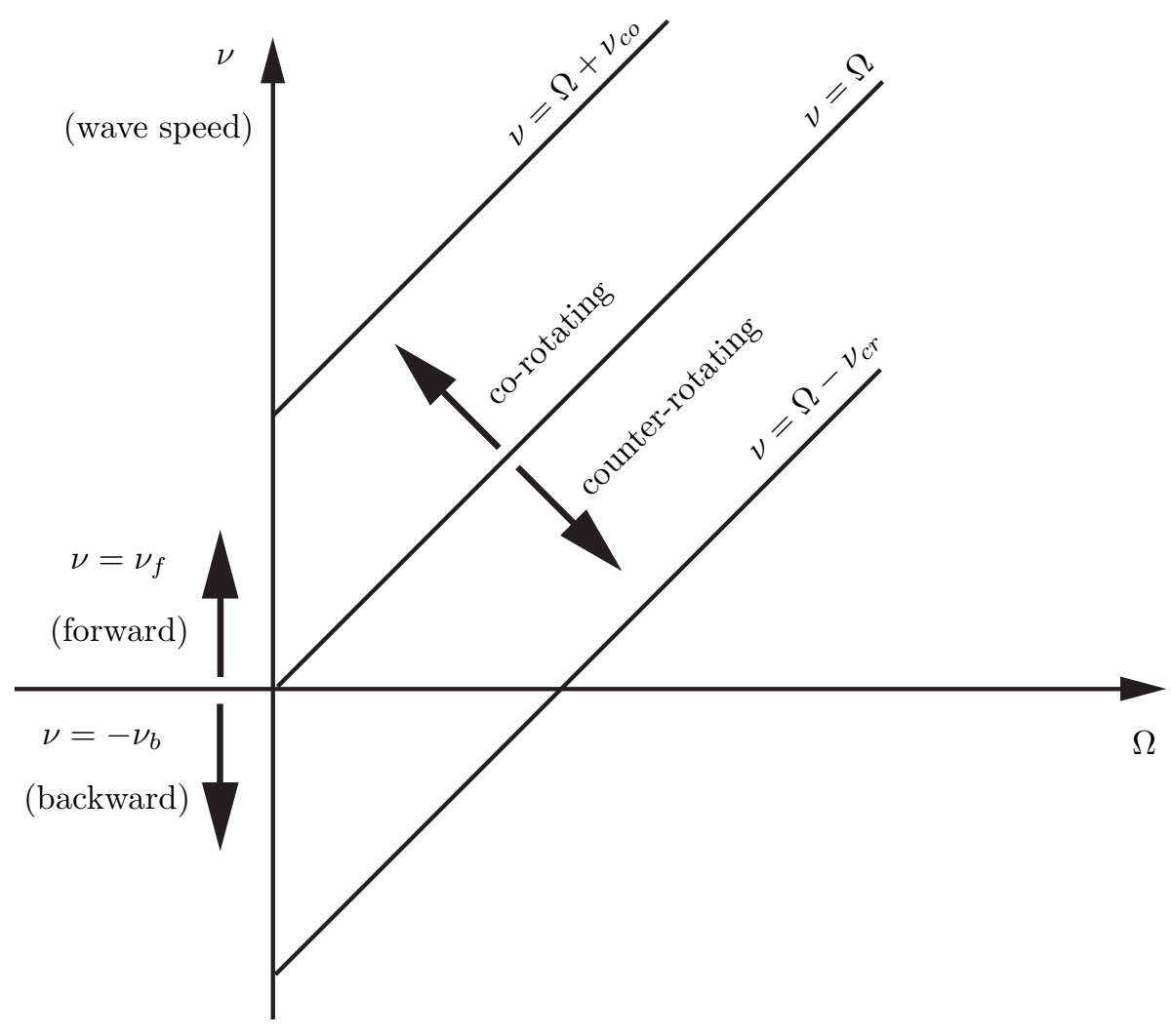

Figure 23: Wave velocity versus rotational velocity diagram explaining directional terms.

direction in a stationary frame $\mathbf{R}_{\mathbf{0}}$. Consistently, a backward wave propagates at speed $\nu_{b}$ in the negative direction (or at speed $-\nu_{b}$ in the positive direction), meaning it propagates at $\nu_{b}$ in a clockwise direction. Consider now a new reference frame $\mathbf{R}$ rotating at speed $\Omega$ with respect to the stationary reference frame $\mathbf{R}_{\mathbf{0}}$. The notion of direction has changed. The wave that propagates in the positive direction with respect to $\mathbf{R}$ is said to be co-rotating at speed $\nu_{c o}$ whereas the one that propagates in the negative direction is said to be counter-rotating at speed $\nu_{c r}$. As drawn in Fig. 23, a counter-rotating wave in a rotating structure can be viewed as a forward traveling wave in $\mathbf{R}_{\mathbf{0}}$ if $\Omega \geq \nu_{c r}$. Depending on the speed of rotation, the counter-rotating wave can change its direction in the stationary reference frame from a backward traveling wave at low rotation speeds to a forward traveling wave at higher rotation speeds. The rotation speed for which the counter-rotating wave is a standing (or stationary) wave in $\mathbf{R}_{\mathbf{0}}$ is called "engine order speed". 
In this paper, it is assumed that the angular velocity of the rotor is always greater than any traveling wave velocity. Therefore, only forward waves, either in the casing or the bladed disk, can be observed in $\mathbf{R}_{\mathbf{0}}$.

\section{Rotating modes}

The term "rotating mode", while visually conspicuous, is rarely used in the literature except in [12]. It expresses the fact that for structures featuring axi-symmetry in general, an eigenanalysis gives rise to $k$-nodal diameter double modes (orthogonal modes with similar $k$-nodal diameter shape and identical frequency) that can be combined with appropriate time dependent functions so that they become $k$-nodal diameter traveling (rotating) waves. These special waves are named "rotating modes" in the present study.

\section{References}

[1] J. G. Marshall, M. Imregun, A review of aeroelasticity methods with emphasis on turbomachinery applications, Journal of Fluids and Structures 10 (3) (1996) 237-267.

[2] C.-W. Lee, Vibration Analysis of Rotors, Kluwer Academic Publishers, Dordrecht ; Boston, 1993.

[3] D. Childs, Turbomachinery Rotordynamics Phenomena, Modeling and Analysis, Wiley, New York, 1993.

[4] A. Muszynska, P. Goldman, Chaotic responses of unbalanced rotor/bearing/ stator systems with looseness or rubs, Chaos, Solitons \& Fractals 5 (1995) 1683-1704.

[5] Y. Choi, Investigation on the whirling motion of full annular rotor rub, Journal of Sound and Vibration 258 (2001) 191-198.

[6] E. Arnoult, Numerical modelling and experimental approach to dynamical contact: application to blades/casing contact in aircraft engines (in French), Ph.D. thesis, Université de Nantes (2000).

[7] P. Schmiechen, Travelling Wave Speed Coincidence, Ph.D. thesis, Imperial College of Science, Technology and Medecine - University of London (1997).

[8] M. Berthillier, J.-P. Mascarell, Vibration of bladed disks: rotor-stator interaction study, (in French), Rapport interne SNECMA YLEC N DR/152/91 (1991).

[9] M. Legrand, B. Peseux, C. Pierre, E. Seinturier, Amélioration de la prédiction de l'interaction rotor/stator dans un moteur d'avion, in: Sixième Colloque National en Calcul des Structures, Tome 1, Giens, France, 2003, pp. 291-298.

[10] S. K. Sinha, Non-linear dynamic response of a rotating radial Timoshenko beam with periodic pulse loading at the free-end, International Journal of Non-Linear Mechanics 40 (2005) $113-149$.

[11] T. Belytschko, W. Liu, B. Moran, Nonlinear Finite Elements for Continua and Structures, John Wiley, Chichester ; New York, 2000.

[12] D. Thomas, Dynamics of rotationally periodic structures, International Journal for Numerical Methods in Engineering 14 (1979) 81-102. 
[13] R. Bladh, Efficient Predictions of the Vibratory Response of Mistuned Bladed Disks by Reduced Order Modeling, Ph.D. thesis, The University of Michigan, Ann Arbor, USA (2001).

[14] P. Davis, Circulant Matrices, second edition, Chelsea Publishing, New York, 1994.

[15] L. Meirovitch, Analytical Methods in Vibrations, Macmillan, New-York, 1967.

[16] W. Leissa, Vibration of Shells, NASA SP 288, Scientific and Technical Information Office, Washington D.C., 1973.

[17] P. Raveendranath, G. Singh, B. Pradhan, Free vibration of arches using a curved beam element based on a coupled polynomial displacement field, Computers \& Structures 78 (2000) 583-590.

[18] T. Laursen, Computational contact and impact mechanics, Springer, Berlin, 2003.

[19] P. Papadopoulos, R. L. Taylor, A simple algorithm for three-dimensional finite element analysis of contact problems, Computers \& Structures 46 (1993) 1107-1118.

[20] K.-J. Bathe, Finite Element Procedures, Prentice-Hall, Englewood Cliffs ; New Jersey, 1996.

[21] J. C. Simo, T. A. Laursen, An augmented lagrangian treatment of contact problems involving friction, Computers \& Structures 42 (1) (1992) 97-116.

[22] P. Chabrand, O. Chertier, F. Dubois, Complementarity methods for multibody friction contact problems in finite deformations, International Journal for Numerical Methods in Engineering 51 (2001) 553-578.

[23] P. Chabrand, F. Dubois, M. Raous, Various numerical methods for solving unilateral contact problems with friction, Mathematical Computer and Modelling 28 (1998) 97-108.

[24] D. Vola, E. Pratt, M. Raous, M. Jean, Consistent time discretization for a dynamical contact problem and complementarity techniques, Revue Européenne des Éléments Finis 7 (1998) 149-162.

[25] N. Carpenter, R. Taylor, M. Katona, Lagrange constraints for transcient finite element surface contact, International Journal for Numerical Methods in Engineering 32 (1991) $103-128$.

[26] M. Yi, J. He, B. Huang, H. Zhou, Friction and wear behavior and abradability of abradable seal coating, Wear 231 (1999) 47-53.

[27] G. S. McNulty, J. J. Decker, B. F. Beacher, S. A. Khalid, The impact of forward swept rotors on tip clearance flows in subsonic axial compressors, Journal of Turbomachinery 126 (4) (2004) 445-454.

[28] D. J. Ewins, The effects of detuning upon the forced vibrations of bladed disks, Journal of Sound and Vibration 9 (1) (1969) 65-79.

[29] T. R. Camp, A study of acoustic resonance in a low speed multistage compressor, Journal of Turbomachinery 121 (1) (1999) 36-43. 